\title{
INVARIANCE KERNELS OF SINGLE-INPUT PLANAR NONLINEAR SYSTEMS*
}

\author{
MANFREDI MAGGIORE ${ }^{\dagger}$, BARRY RAWN $^{\ddagger}$, AND PETER LEHN ${ }^{\dagger}$
}

\begin{abstract}
The problem of determining invariance kernels for planar single-input nonlinear systems is considered. If $K$ is a closed set, its invariance kernel is the largest subset of $K$ with the property of being positively invariant for arbitrary measurable input signals. It is shown that the boundary of the invariance kernel is a concatenation of solutions of two so-called extremal vector fields. Moreover, only the solutions through a finite number of special points are of interest. This result makes it possible to devise an algorithm which determines the invariance kernel of a simply connected set in a finite number of steps.
\end{abstract}

Key words. invariance and viability kernels, extremal vector fields, switched systems, differential inclusions

AMS subject classifications. Primary, 93C10; Secondary, 93B03, 93C30

DOI. $10.1137 / 100804784$

1. Introduction. In this paper we consider the planar system

$$
\Sigma: \dot{x}=\lambda(t) f_{1}(x)+[1-\lambda(t)] f_{2}(x),
$$

where $f_{1}, f_{2}: \mathbb{R}^{2} \rightarrow \mathbb{R}^{2}$ are two $C^{1}$ planar vector fields and $\lambda(t)$ is a signal in the class $\mathcal{U}$ of measurable functions $\mathbb{R} \rightarrow[0,1]$. We make a number of generic assumptions which are listed in section 3 . Viewing $\lambda(t)$ as a control signal, $\Sigma$ is a control-affine system. Conversely, any control-affine system $\dot{x}=f(x)+g(x) u$ with scalar compactvalued controls $u \in\left[u_{\min }, u_{\max }\right] \subset \mathbb{R}$ can be expressed in the form (1.1) by letting $f_{1}(x)=f(x)+g(x) u_{\min }, f_{2}(x)=f(x)+g(x) u_{\max }$ and expressing $u(t)=\lambda(t) u_{\min }+$ $(1-\lambda(t)) u_{\max }$.

The objective of this paper is the characterization of the invariance kernel of a closed set $K$, defined next.

Definition 1.1. Let $K \subset \mathbb{R}^{2}$ be a closed set. $K$ is positively invariant (or strongly invariant) for $\Sigma$ if for all $\lambda(t) \in \mathcal{U}$ and all $x_{0} \in K$, the solution of $\Sigma$ with initial condition $x(0)=x_{0}$ remains in $K$ for all $t \geq 0$. The invariance kernel $K^{\star}$ of $K$ for system $\Sigma$ is the maximal positively invariant subset of $K$.

The invariance kernel of a closed set is closed, for if a set is positively invariant its closure is positively invariant as well. The notion of positive invariance (or strong invariance) of $K$ defined above requires all solutions of $\Sigma$ originating in $K$ to remain in $K$ for all positive time. In contrast, $K$ is said to be weakly invariant, or viable, for $\Sigma$ if for all $x_{0} \in K$, at least one solution of $\Sigma$ through $x_{0}$ remains in $K$ for all $t \geq 0$. Accordingly, the viability kernel of $K$ for system $\Sigma$ is the maximal subset of $K$ with the property of being viable for $\Sigma$.

${ }^{*}$ Received by the editors August 9, 2010; accepted for publication (in revised form) December 27, 2011; published electronically April 26, 2012.

http://www.siam.org/journals/sicon/50-2/80478.html

$\dagger$ Department of Electrical and Computer Engineering, University of Toronto, 10 King's College Road, Toronto, ON, M5S 3G4, Canada (maggiore@control.utoronto.ca, lehn@ecf.utoronto.ca). These authors were supported by the Natural Sciences and Engineering Research Council of Canada (NSERC).

$\ddagger$ Department of Electrical Sustainable Energy, Delft University of Technology, Mekelweg 4, 2628 CD Delft, The Netherlands (b.g.rawn@tudelft.nl). This research was carried out while this author was with the Department of Electrical and Computer Engineering, University of Toronto.

1012 
The theory of viability and invariance kernels was developed by Aubin and coworkers in the general setting of differential inclusions. See Aubin's book [3] for an overview of the subject. (See also the review paper [7].) To relate our problem statement to Aubin's general theory, we remark that system (1.1) can be associated with the differential inclusion

$$
\Sigma_{I}: \dot{x} \in F(x):=\operatorname{co}\left\{f_{1}(x), f_{2}(x)\right\} \text { a.e., }
$$

where co $\left\{f_{1}(x), f_{2}(x)\right\}$ denotes the convex hull of $f_{1}(x)$ and $f_{2}(x)$. By Filippov's selection lemma (see [2]), trajectories of $\Sigma_{I}$ in (1.2) are solutions of $\Sigma$ corresponding to suitable selections $\lambda(t) \in \mathcal{U}$. Conversely, it is obvious that solutions of $\Sigma$ with $\lambda(t) \in \mathcal{U}$ are trajectories of $\Sigma_{I}$. Therefore, there is a one-to-one correspondence between solutions of $\Sigma$ and those of $\Sigma_{I}$. Owing to this correspondence, determining the invariance kernel of $K$ for $\Sigma$ or for $\Sigma_{I}$ is the same, and all results concerning invariance kernels of differential inclusions apply directly to $\Sigma$ in (1.1).

Applications of Aubin's theory abound and are found in fields as diverse as ecology, climatology, biology, and economics $[8,14,15,22]$. Recently, invariance kernels were used in [25] to define and quantify a notion of stability margin for wind turbines. In control theory, viability kernels often appear in the form of maximal controlled invariant sets. The investigation of such sets originated with work on geometric linear systems theory by Wonham and Morse [29] and Basile and Marro [6] and culminated in the nonlinear setting of control-affine systems with the zero dynamics algorithm of Byrnes and Isidori [18]. On the other hand, in control theory invariance kernels are associated with notions of robustness (e.g., [24, 19]).

Many of the numerical methods for approximating viability kernels (and invariance kernels after some adaptation) available in the literature are based on a scheme, called the viability kernel algorithm, which originated in the work of Frankowska and Quincampoix [17] and Saint-Pierre [27]. The scheme in question involves discretizing the differential inclusion in time and forming a covering of $K$. Successive refinements of the viability kernel of $K$ are then computed in a way similar to the algorithm of Wonham and Morse and Basile and Marro. In [26], Rieger gave convergence estimates for this algorithm. In [20], Labinaz and Guay applied the algorithm to a class of hybrid systems. Recently, Broucke and Turriff [10] gave an explicit characterization of the viability kernel for a class of control-affine systems when $K$ is the sublevel set of a smooth function. They were able to show that under certain conditions the viability kernel is a sublevel set of a hitting time function.

Letting $\mathcal{U}^{ \pm} \subset \mathcal{U}$ be the class of measurable functions $\mathbb{R} \rightarrow\{0,1\}$ and taking $\lambda(t) \in \mathcal{U}^{ \pm}, \Sigma$ becomes a switched system. In this context, the invariance kernel $K^{\star}$ of $K$ is the maximal subset of $K$ with the property that for any switching signal $\lambda(t) \in \mathcal{U}^{ \pm}$, solutions of $\Sigma$ originating in $K^{\star}$ remain in $K^{\star}$ in positive time. It turns out that the invariance kernels one obtains by letting either $\lambda(t) \in \mathcal{U}$ or $\lambda(t) \in \mathcal{U}^{ \pm}$in Definition 1.1 coincide. The results in this paper are therefore relevant to the literature on switched systems. In this context, a problem which attracted considerable attention is that of finding conditions under which the origin of the switched system is globally uniformly asymptotically stable (GUAS). See, for instance, [1] for switched linear systems and [21] for a nonlinear generalization. In the case of planar systems, Boscain, Charlot, and Sigalotti in [9] provided some sufficient and some necessary conditions for the GUAS property to hold which rely on the geometry of the set where the vector fields $f_{1}$ and $f_{2}$ are parallel. This set plays an important role in this paper as well and is used to define two extremal vector fields, having the property that 
their solutions are the trajectories of $\Sigma$ with minimum and maximum slope. These fields have been independently studied in relation to attainable sets by Baitman [5], Butenina [11], and Davydov [13].

This paper makes two main contributions. The first, in Theorem 7.1, is a characterization of the boundary of the invariance kernel for the planar system $\Sigma$ in terms of integral curves of extremal vector fields through a finite number of special points. The concatenation of such integral curves must obey precise rules in order to form a feasible boundary of $K^{\star}$. The second main contribution of this paper is an algorithm which exploits the finiteness of special points and the concatenation rules to determine the invariance kernel in a finite number of steps. In this paper we assume, among other things, that $K$ is a simply connected set, but our algorithm can be adapted to the situation when $K$ is not simply connected.

The paper is organized as follows. Section 2 presents various preliminary definitions, including those of the collinearity set and the extremal vector fields. Section 3 contains the assumptions used throughout the paper. Section 4 reviews properties of solutions of the extremal vector fields, including existence and uniqueness, and continuity of their flow maps. The relationship between solutions of extremal vector fields and the boundary of the invariance kernel is established in section 5, and the concatenation rules that integral curves of extremal vector fields must obey in order to form $\partial K^{\star}$ are presented in section 6 . The characterization of $\partial K^{\star}$ is presented in section 7. The algorithm to determine $K^{\star}$ is introduced in section 8 and rigorously justified in section 9. Finally, section 10 contains an example.

Throughout this paper we use the following notation. If $S \subset \mathbb{R}^{2}, S^{c}$ denotes the complement of $S, S^{c}=\mathbb{R}^{2} \backslash S$. The notation $\langle\cdot, \cdot\rangle$ is used to denote the Euclidean inner product. Finally, int $S$ denotes the interior of the set $S$.

2. Preliminary definitions. We now present the basic notions used in the characterization of invariance kernels. Let

$$
\mathcal{R}^{+}=\left\{x \in \mathbb{R}^{2}: \operatorname{det}\left[f_{1}(x) f_{2}(x)\right]>0\right\}, \quad \mathcal{R}^{-}=\left\{x \in \mathbb{R}^{2}: \operatorname{det}\left[f_{1}(x) f_{2}(x)\right]<0\right\} .
$$

$\mathcal{R}^{+}$and $\mathcal{R}^{-}$are open sets. In $\mathcal{R}^{+}, f_{2}$ points to the left-hand side of $f_{1}$, while in $\mathcal{R}^{-}$ $f_{2}$ points to the right-hand side of $f_{1}$. This is illustrated in Figure 2.1.

DEFINITION 2.1. The extremal vector fields $f_{R}(x)$ and $f_{L}(x)$ are defined as

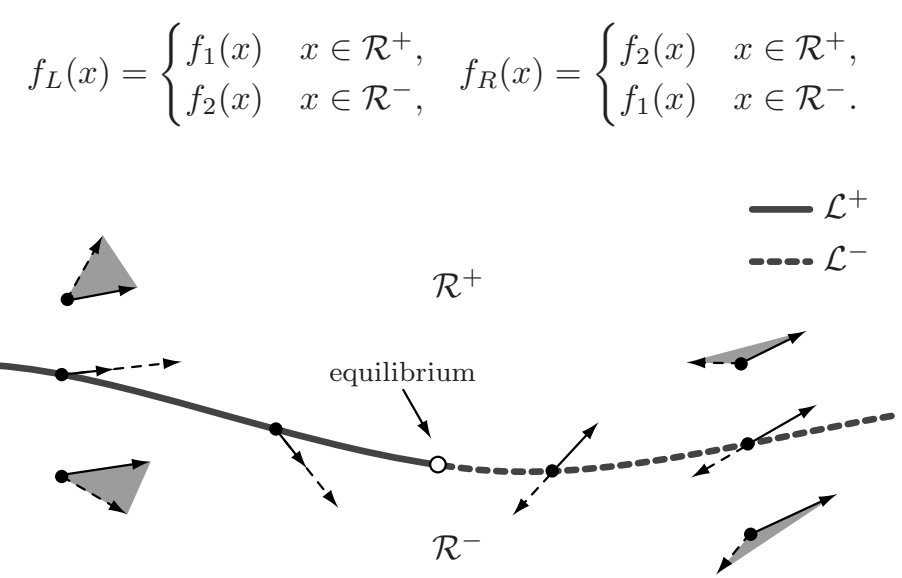

FIG. 2.1. An illustration of sets $\mathcal{R}^{+}, \mathcal{R}^{-}, \mathcal{L}^{+}, \mathcal{L}^{-}$. The vector field $f_{1}$ is depicted in solid arrows, while $f_{2}$ is depicted in dashed arrows. 


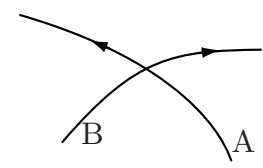

Fig. 2.2. Arc A crosses arc B "leftward," while arc B crosses arc A "rightward."

The solutions ${ }^{1}$ at time $t$ of the extremal vector fields $f_{L}$ and $f_{R}$ are called extremal solutions and are denoted by $\phi_{L}\left(t, x_{0}\right)$ and $\phi_{R}\left(t, x_{0}\right)$, respectively. The images of extremal solutions on the plane are called extremal arcs. In particular, the L-arc (resp., R-arc) through $x_{0}$ is the image of the map $t \mapsto \phi_{L}\left(t, x_{0}\right)$ (resp., $t \mapsto$ $\left.\phi_{R}\left(t, x_{0}\right)\right)$ for $t$ ranging over some interval over which the map is defined.

DeFINITION 2.2. A connected subset of $\partial K$ along which both $f_{1}$ and $f_{2}$ point inside of $K$ or are tangent to $\partial K$ is said to be an invariant arc of $\partial K$. Each endpoint of an invariant arc of $\partial K$ is called a $\boldsymbol{t}^{\partial}$ point.

We give an orientation to extremal arcs and invariant arcs of $\partial K$ as follows. We give $\partial K$ a positive orientation so that a point moving along $\partial K$ finds the interior of $K$ to its left-hand side. The orientation of extremal arcs is induced by the time parametrization of the corresponding extremal solutions, so that the orientation indicates the direction of increasing time. The notion of orientation of arcs allows us to say, for instance, that arc A crosses arc B leftward to describe the situation depicted in Figure 2.2.

Definition 2.3. Suppose that $\bar{x}$ is an equilibrium of $f_{1}$ (resp., $f_{2}$ ). An extremal arc through $\bar{x}$ is said to be an equilibrium extremal arc through $\bar{x}$ if on a neighborhood of $\bar{x}$ it coincides with an $f_{1}$ arc (resp., $f_{2}$ arc). If, instead, the extremal arc coincides with an $f_{2}$ arc (resp., $f_{1}$ arc) in a neighborhood of $\bar{x}$, then it is said to be a nonequilibrium extremal arc through $\bar{x}$.

DEFINITION 2.4. We define the collinearity set $\mathcal{L}$ as

$$
\mathcal{L}=\left\{x \in \mathbb{R}^{2}: \operatorname{det}\left[f_{1}(x) f_{2}(x)\right]=0\right\}
$$

and the sets $\mathcal{L}^{+}=\left\{x \in \mathcal{L}:\left\langle f_{1}(x), f_{2}(x)\right\rangle>0\right\}, \mathcal{L}^{-}=\left\{x \in \mathcal{L}:\left\langle f_{1}(x), f_{2}(x)\right\rangle<0\right\}$.

On $\mathcal{L}, f_{1}$ and $f_{2}$ are collinear. On $\mathcal{L}^{-}, f_{1}$ and $f_{2}$ are antiparallel. Points in $\mathcal{L}$ that are neither in $\mathcal{L}^{+}$nor in $\mathcal{L}^{-}$are equilibria of $f_{1}$ or $f_{2}$. This is illustrated in Figure 2.1. The set $\mathcal{L}$ is closed and in this paper we will assume (see section 3 ) that it is a one-dimensional embedded submanifold. The extremal vector fields $f_{L}, f_{R}$ are discontinuous on $\mathcal{L}$. The existence and uniqueness of extremal solutions is discussed in section 4.

Definition 2.5. A point $p$ in $\mathcal{L}^{-}$is called a $\boldsymbol{t}^{-}$point if the trajectories of $f_{1}(x)$ and $f_{2}(x)$ through $p$ remain in the closure of either $\mathcal{R}^{+}$or $\mathcal{R}^{-}$for some time interval containing $t=0$ (i.e., $\left\langle f_{1}, f_{2}\right\rangle$ has constant sign along the trajectories of $f_{1}(x)$ and $f_{2}(x)$ through $p$ for small time).

The definitions of $t^{-}, t^{\partial}$ points, and invariant arcs of $\partial K$ are illustrated in Figure 2.3. If $\partial K$ is differentiable in a neighborhood of a $t^{\partial}$ point, then at least one of the vector fields $f_{1}, f_{2}$ must be tangent to $\partial K$ at the $t^{\partial}$ point.

DEFInITION 2.6. The attainable set $\mathcal{A}\left(x_{0}, t\right)$ of $\Sigma$ from $x_{0}$ at time $t$ is

$$
\mathcal{A}\left(x_{0}, t\right)=\left\{x(t): x(\cdot) \text { is a solution of } \Sigma \text { with } x(0)=x_{0} \text { for some } \lambda(\cdot) \in \mathcal{U}\right\} \text {. }
$$

\footnotetext{
${ }^{1}$ By a solution of an extremal vector field we mean an absolutely continuous function $x(t)$ : $(a, b) \rightarrow \mathbb{R}^{2}$ which satisfies the differential equation associated with the extremal vector field for almost all $t \in(a, b)$.
} 

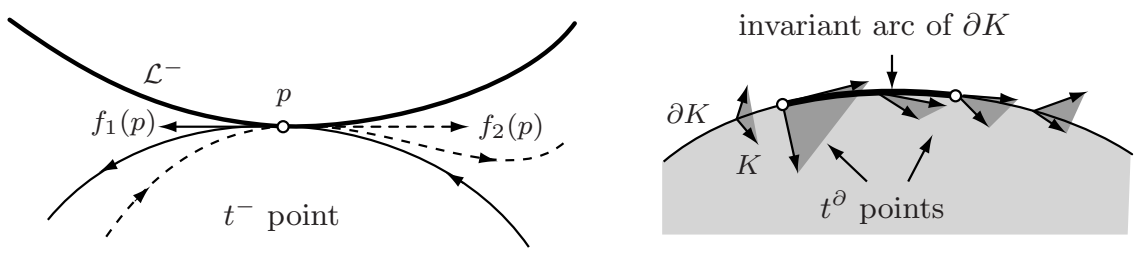

FIG. 2.3. An illustration of $t^{-}, t^{\partial}$ points, and invariant arcs of $\partial K$.

By Theorem 1 in section 2.8 of [16], the set-valued map $\left(x_{0}, t\right) \mapsto \mathcal{A}\left(x_{0}, t\right)$ is upper semicontinuous. Moreover, $\mathcal{A}\left(x_{0}, t\right)$ is compact and nonempty [12].

Remark 1. By the definition of $f_{L}$ and $f_{R}$, for each $x \in \mathbb{R}^{2} \backslash \mathcal{L}$ and all $\lambda \in(0,1)$, the vector $\lambda f_{1}(x)+(1-\lambda) f_{2}(x)$ points to the left-hand side of $f_{L}(x)$ and to the right-hand side of $f_{R}(x)$. Moreover, when $\lambda$ is 0 or 1 , the vector $\lambda f_{1}(x)+(1-\lambda) f_{2}(x)$ is tangent to either $f_{L}(x)$ or $f_{R}(x)$. Therefore, all solutions of $\Sigma$ in $\mathbb{R}^{2} \backslash \mathcal{L}$ are either tangent to or cross R-arcs rightward and L-arcs leftward. In particular, R-arcs (resp., L-arcs) in $\mathbb{R}^{2} \backslash \mathcal{L}$ are either tangent to or cross L-arcs (resp., R-arcs) leftward (resp., rightward). It can be shown that the above statement is true not just for arcs in $\mathbb{R}^{2} \backslash \mathcal{L}$ but in the entire $\mathbb{R}^{2}$ except at equilibria of $f_{1}$ or $f_{2}$. Extremal arcs cannot self-intersect at points other than equilibria of $f_{1}$ and $f_{2}$.

3. Standing assumptions. Throughout this paper we assume that $K$ is simply connected and its boundary is a $C^{1}$ Jordan curve. Additionally, we make these assumptions:

(i) The set $\mathcal{L}$ is a one-dimensional embedded submanifold, i.e., it is the union of a countable number of disjoint regular curves.

(ii) There is a finite number of $t^{-}$points in $K$, and there is at most a finite number of points on $\partial K$ at which either $f_{1}$ or $f_{2}$ are tangent to $\partial K$.

(iii) The equilibria of $f_{1}$ and $f_{2}$ in $K$ are hyperbolic (implying that all equilibria are isolated) and the linearization at each equilibrium has distinct eigenvalues. Moreover, none of the equilibria of $f_{1}$ is an equilibrium of $f_{2}$.

(iv) No equilibria of $f_{1}$ and $f_{2}$ lie on $\partial K$.

(v) The slow manifolds of nodes (stable or unstable) of $f_{1}$ and $f_{2}$ are not tangent to $\mathcal{L}$.

(vi) No $t^{\partial}$ points lie on $\mathcal{L}$.

(vii) There is a finite number of points on $\mathcal{L}^{+} \cap K$, where $f_{1}$ and $f_{2}$ are tangent to $\mathcal{L}^{+}$.

(viii) There is at most a finite number of closed extremal arcs in $K$.

Remark 2. Assumptions (i)-(vii) are $C^{1}$-generic. Assumptions (iii)-(vii) could be relaxed, but they are made to avoid the need for special cases and to simplify the presentation. Finally, the assumption that $\partial K$ is differentiable is made only to simplify the arguments of the proofs. All the results presented in this paper hold in the case when $\partial K$ is not differentiable. Note, indeed, that our definition of $t^{\partial}$ point does not rely on the differentiability of $\partial K$.

4. Properties of extremal solutions. The extremal vector fields $f_{L}$ and $f_{R}$ are discontinuous on $\mathcal{L}$. Issues of existence and uniqueness of solutions of vector fields of this kind have been extensively investigated by Filippov [16]. Solutions of $f_{L}$ and $f_{R}$ exist everywhere on the plane. The next two lemmas discuss issues of nonuniqueness and continuity of the solution maps $\phi_{L}$ and $\phi_{R}$. 


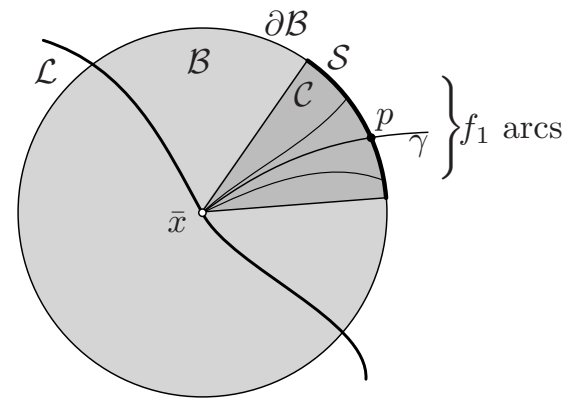

FIG. 4.1. Illustration of Lemma 4.3.

Lemma 4.1. Extremal solutions of $\Sigma$ exist through each $x_{0} \in \mathbb{R}^{2}$. Locally near each point $x_{0} \in \mathbb{R}^{2}$, there is only one $L$-arc and one $R$-arc through $x_{0}$, except in the following cases:

(i) If $x_{0} \in \mathcal{L}^{-}$and $x_{0}$ is not a $t^{-}$point, then through $x_{0}$ there are either two $L$-arcs which converge to and two $R$-arcs which diverge from $x_{0}$, or two L-arcs which diverge from and two $R$-arcs that converge to $x_{0}$. In a neighborhood of $x_{0}$, the two L-arcs (resp., $R$-arcs) coincide with an arc of $f_{1}$ in $\mathcal{R}^{+}$(resp., in $\mathcal{R}^{-}$) and and an arc of $f_{2}$ in $\mathcal{R}^{-}$(resp., in $\mathcal{R}^{+}$).

(ii) If $x_{0}$ is an equilibrium of $f_{1}$ or $f_{2}$, then there is one nonequilibrium extremal arc through $x_{0}$ and several, possibly infinite, equilibrium extremal arcs through $x_{0}$.

Proof. Part (i) of the lemma follows from Corollary 2 on p. 108 of [16] and the fact that there is no sliding motion on $\mathcal{L}$ in a neighborhood of $x_{0}$, because the vector fields $f_{1}, f_{2}$ are antiparallel and not tangent to $\mathcal{L}$ at $x_{0}$. The fact that if $x_{0}$ is a $t^{-}$ point or a point on $\mathcal{L}^{+}, f_{1}$ arcs and $f_{2}$ arcs through $x_{0}$ are locally unique follows from Lemma 2 on p. 107 of [16]. The fact that solutions through points in $\mathcal{R}^{+} \cup \mathcal{R}^{-}$are locally unique is obvious since $f_{L}$ and $f_{R}$ are $C^{1}$ on this set.

LEMma 4.2. Suppose that $x_{0} \notin \mathcal{L}^{-}$and $x_{0}$ is not an equilibrium of $f_{1}$ or $f_{2}$. Suppose that the unique solution $x(t)$ of $f_{L}$ (resp., $f_{R}$ ) through $x_{0}$ is defined on $[0, T] \subset$ $\mathbb{R}$ and such that for all $t \in[0, T], x(t) \notin \mathcal{L}^{-}$and $x(t)$ is not an equilibrium of $f_{1}$ or $f_{2}$. Then, there exists a neighborhood $U$ of $x_{0}$ such that the map $\phi_{L}\left(t, x_{0}\right)$ (resp., $\left.\phi_{R}\left(t, x_{0}\right)\right)$ is continuous on $[0, T] \times U$.

Proof. The lemma follows directly from the fact that the solution $x(t)$ of $f_{L}$ (resp., $\left.f_{R}\right)$ is unique on $[0, T]$ and from Theorem 2, p. 90, of [16].

We conclude this section with a characterization of equilibrium extremal arcs in a neighborhood of a node (stable or unstable). Before stating the result, we recall that if the linearization of a planar vector field at a node has two distinct eigenvalues, then the fast manifold of the node is the invariant manifold of the vector field associated with the eigenvalue which has the largest absolute value, while the slow manifold is associated with the eigenvalue that has smallest absolute value.

Lemma 4.3. Suppose that an L-arc (resp., $R$-arc) $\gamma$ is an equilibrium extremal arc through a node $\bar{x}$, and that, in a neighborhood of $\bar{x}, \gamma$ does not coincide with the fast manifold of $\bar{x}$. Then, there exists a ball $\mathcal{B}$ centered at $\bar{x}$ and a circle segment $\mathcal{S} \subset \partial \mathcal{B}$ with a unique intersection point $p=\mathcal{S} \cap \gamma$ such that all L-arcs (resp., $R$-arcs) through $\mathcal{S}$ remain in $\mathcal{B}$ in positive or negative time and are equilibrium extremal arcs.

Proof. We will prove the lemma assuming, without loss of generality, that $\bar{x}$ is a stable node of $f_{1}$. The proof when $\bar{x}$ is an unstable node is analogous. The various constructions that follow are illustrated in Figure 4.1. Since $\gamma$ is an equilibrium 
extremal arc, there exists a ball $\mathcal{B}$ centered at $\bar{x}$ such that $\hat{\gamma}:=\gamma \cap \mathcal{B}$ is an $f_{1}$ arc. By assumption, $\hat{\gamma}$ is not the fast manifold of $\bar{x}$, and therefore it must be tangent at $\bar{x}$ to the slow manifold of $\bar{x}$. By the standing assumption (v) in section 3 , the slow manifold in question is not tangent to $\mathcal{L}$ at $\bar{x}$, and therefore $\hat{\gamma}$ is not tangent to $\mathcal{L}$ at $\bar{x}$ either. Thus, $\mathcal{B}$ can be chosen small enough that there exists a sector $\mathcal{C}$ of $\mathcal{B}$ which is positively invariant for $f_{1}$ and such that $\mathcal{C} \cap \mathcal{L}=\{\bar{x}\}$. By the positive invariance of $\mathcal{C}$, we can assume that $f_{1}$ is transversal to $\partial \mathcal{B} \cap \mathcal{C}$ (if not, we could make $\mathcal{B}$ smaller), implying that $\partial \mathcal{B} \cap \gamma$ is a unique point $p$. Letting $\mathcal{S}=\partial \mathcal{B} \cap \mathcal{C}$, all $f_{1}$ arcs through points in $\mathcal{S}$ remain in $\mathcal{C}$, and hence in $\mathcal{B}$, for all positive time and do not intersect $\mathcal{L}$, except at the equilibrium $\bar{x}$. Therefore, $f_{1}$ arcs and L-arcs through points in $\mathcal{S}$ coincide in positive time, proving that all L-arcs through points in $\mathcal{S}$ are equilibrium extremal arcs.

5. Extremal arcs and boundary of the invariance kernel. The significance of extremal arcs, as pertains to the determination of invariance kernels, is that they form the boundary of attainable sets of $\Sigma$, as shown in the next lemma. Thus, extremal arcs delimit bundles of arcs of $\Sigma$ through points in $\mathbb{R}^{2}$ resulting from arbitrary choices of $\lambda(t) \in \mathcal{U}$, a property illustrated in Figure 5.1. This feature of extremal arcs, together with the so-called barrier property presented in Proposition 5.2 below, will be used in Proposition 5.3 to establish a relationship between extremal arcs and boundaries of invariance kernels. Before stating the lemma, we recall that $\Sigma$ is said to be smalltime locally controllable (STLC) from $x_{0}$ if, for all $T>0, x_{0}$ lies in the interior of $\mathcal{A}\left(x_{0},[0, T]\right)$.

LEMMA 5.1. Let $x_{0} \in \mathbb{R}^{2}$ be such that $\Sigma$ is not STLC from $x_{0}$. Suppose that for some $T>0$, a solution $x(t)$ of $\Sigma$ with initial condition $x_{0}$ has the property that $x(t) \in \partial \mathcal{A}\left(x_{0}, t\right)$ for all $t \in[0, T]$ and that $\Sigma$ is not $S T L C$ from $x(t)$ for all $t \in[0, T]$. Then, $x(t)$ is a concatenation of extremal solutions.

Proof. We claim that for $x_{0}$ as in the statement of the lemma, there exists $\bar{t} \in(0, T]$ such that $\bigcup_{t \in[0, \bar{t}]} \partial \mathcal{A}\left(x_{0}, t\right)$ is the union of arcs of $f_{1}$ and $f_{2}$, and hence also of extremal arcs. This property will then imply that on the time interval $[0, \bar{t}]$ $x(t)$ is the union of extremal arcs since for all $t \in[0, \bar{t}], x(t) \in \bigcup_{t \in[0, \bar{t}]} \partial \mathcal{A}\left(x_{0}, t\right)$. The repeated application of this claim yields the required result on the entire interval $[0, T]$. In order to prove the claim, we observe that the plane is partitioned by the sets

$$
\mathbb{R}^{2}=\mathcal{R}^{+} \cup \mathcal{R}^{-} \cup \mathcal{L}^{+} \cup \mathcal{L}^{-} \cup\left\{\text { equilibria of } f_{1} \text { or } f_{2}\right\}
$$

and therefore there are four cases of interest.

Case 1. $x_{0} \in \mathcal{R}^{+} \cup \mathcal{R}^{-}$. Since $\mathcal{R}^{+} \cup \mathcal{R}^{-}$is an open set and $\left(x_{0}, t\right) \mapsto \mathcal{A}\left(x_{0}, t\right)$ is upper semicontinuous, there exists $\bar{t}>0$ such that $\mathcal{A}\left(x_{0},[0, \bar{t}]\right) \subset \mathcal{R}^{+} \cup \mathcal{R}^{-}$. Since $\mathcal{R}^{+}$ and $\mathcal{R}^{-}$are disjoint and $\mathcal{A}\left(x_{0},[0, \bar{t}]\right)$ is connected, the set $\mathcal{A}\left(x_{0},[0, \bar{t}]\right)$ is contained in

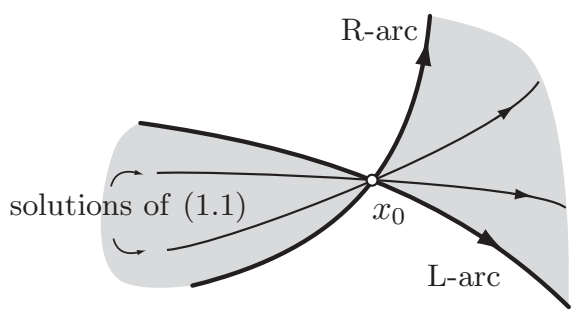

FIG. 5.1. Bounding property of extremal trajectories. 


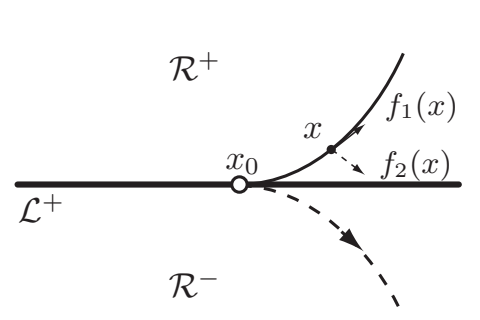

(a)

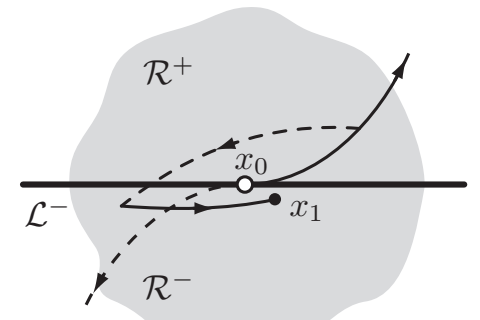

(b)

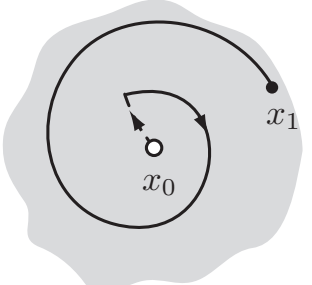

(c)

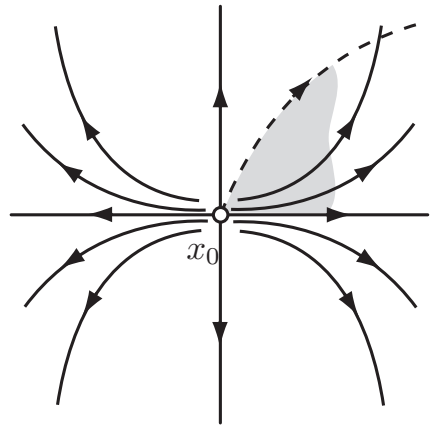

(d)

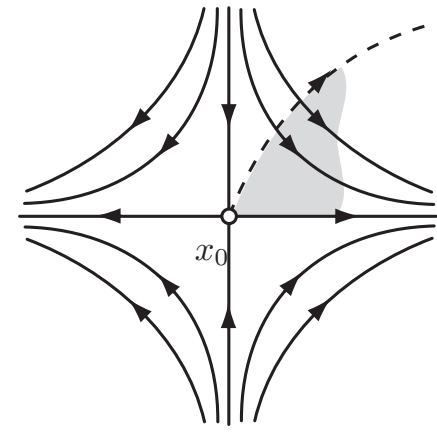

(e)

Fig. 5.2. Illustration of arguments in the proof of Lemma 5.1. Part (a) refers to case 2 in the proof, part (b) refers to case 3 , and parts (c)-(e) refer to case 4 . Solid lines indicate $f_{1}$ arcs, while dashed lines indicate $f_{2}$ arcs. Shaded areas indicate attainable sets $\mathcal{A}\left(x_{0},[0, \bar{t}]\right)$ for small $\bar{t}>0$.

one of them, say, $\mathcal{R}^{+}$. On $\mathcal{R}^{+}$, all arcs of $\Sigma$ cross arcs of $f_{1}$ leftward and those of $f_{2}$ rightward. Therefore, $\bigcup_{t \in[0, \bar{t}]} \partial \mathcal{A}\left(x_{0}, t\right)$ is the union of arcs of $f_{1}$ and $f_{2}$ through $x_{0}$, proving the claim in this case.

Case 2. $x_{0} \in \mathcal{L}^{+}$. In this case, $f_{1}\left(x_{0}\right), f_{2}\left(x_{0}\right)$ are parallel. Suppose that the arc of $f_{1}$ through $x_{0}$ enters $\mathcal{R}^{+}$. Then, the arc of $f_{2}$ through $x_{0}$ must also enter $\mathcal{R}^{+}$. If it didn't, by the continuity of $f_{2}$ there would be points in $\mathcal{R}^{+}$near $x_{0}$ at which $f_{2}$ does not point to the left-hand side of $f_{1}$, as illustrated in Figure 5.2(a), which contradicts the fact that $x_{0} \in \mathcal{R}^{+}$. We thus have that there exists $\bar{t}>0$ such that $\mathcal{A}\left(x_{0},(0, \bar{t}]\right) \subset \mathcal{R}^{+}$. By the same reasoning of case $1, \bigcup_{t \in[0, \bar{t}]} \partial \mathcal{A}\left(x_{0}, t\right)$ is the union of arcs of $f_{1}$ and $f_{2}$ through $x_{0}$, proving the claim for this case. The argument for the case when the arc of $f_{1}$ through $x_{0}$ enters $\mathcal{R}^{-}$is analogous. Finally, consider the situation when for some $\bar{t}>0$, the solution of $f_{1}$ through $x_{0}$ remains in $\mathcal{L}^{+}$for all $t \in[0, \bar{t}]$. Then, since on $\mathcal{L}^{+} f_{1}$ and $f_{2}$ are parallel, all solutions of $\Sigma$ through $x_{0}$ remain in $\mathcal{L}^{+}$for some time, without loss of generality $\bar{t}$, and so $\bigcup_{t \in[0, \bar{t}]} \partial \mathcal{A}\left(x_{0}, t\right)$ is an arc of $f_{1}$ and $f_{2}$.

Case 3. $x_{0} \in \mathcal{L}^{-}$. In this case, $f_{1}\left(x_{0}\right), f_{2}\left(x_{0}\right)$ are antiparallel. Suppose that the $f_{1}$ arc through $x_{0}$ enters $\mathcal{R}^{+}$while the $f_{2}$ arc through $x_{0}$ enters $\mathcal{R}^{-}$, or vice versa. This situation occurs whenever $x_{0}$ is not a $t^{-}$point. Figure 5.2(b) illustrates how any point $x_{1}$ in a neighborhood of $x_{0}$ can be reached from $x_{0}$ through a concatenation of $f_{1}$ and $f_{2}$ arcs. In fact, it can be shown that in this case $\Sigma$ is STLC from $x_{0}$. This case, therefore, cannot occur for $x_{0}$ satisfying the hypotheses of the lemma. Next, the situation when both the $f_{1}$ and $f_{2}$ arcs enter either $\mathcal{R}^{+}$or $\mathcal{R}^{-}$is analogous to case 1 , for which the claim was shown to hold. Next, if the solutions of $f_{1}$ and $f_{2}$ through $x_{0}$ remain in $\mathcal{L}^{-}$for some time, then all solutions of $\Sigma$ through $x_{0}$ remain on $\mathcal{L}^{-}$for 


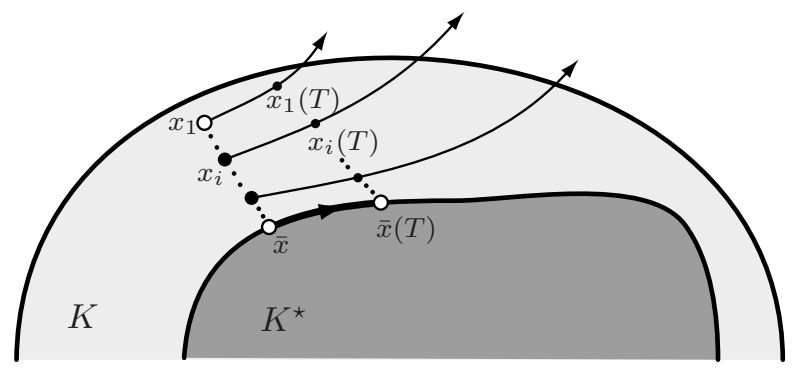

FIG. 5.3. Illustration of construction in the proof of Proposition 5.2.

some time, and thus for some $\bar{t}>0 \bigcup_{t \in[0, \bar{t}]} \mathcal{A}\left(x_{0}, t\right)$ is the concatenation of two arcs of $f_{1}$ and $f_{2}$. The last case left is when one solution through $x_{0}$ of $f_{1}$ or $f_{2}$ remains on $\mathcal{L}^{-}$for some time, while the solution of the other field enters $\mathcal{R}^{+}$or $\mathcal{R}^{-}$. This situation is analogous to the case when both arcs of $f_{1}$ and $f_{2}$ enter $\mathcal{R}^{+}$or $\mathcal{R}^{-}$.

Case 4. $x_{0}$ is an equilibrium of $f_{1}$ or $f_{2}$. Suppose, without loss of generality, that $f_{1}\left(x_{0}\right)=0$ (and therefore $f_{2}\left(x_{0}\right) \neq 0$ ). If $x_{0}$ is a focus (stable or unstable) of $f_{1}$, then it is easy to see that $\Sigma$ is STLC from $x_{0}$, and hence $x_{0}$ violates the hypotheses of the lemma. Figure 5.2(c) illustrates how a generic point $x_{1}$ in a neighborhood of $x_{0}$ can be reached in small time from $x_{0}$ in the case when $x_{0}$ is an unstable focus. Hence, the only cases of interest are when $x_{0}$ is a node (stable or unstable) or a saddle point. In both cases, for sufficiently small $\bar{t}>0, \bigcup_{t \in[0, \bar{t}]} \partial \mathcal{A}\left(x_{0}, t\right)$ is a region delimited by the $f_{2}$ arc through $x_{0}$ and an $f_{1}$ arc, as illustrated for special cases in Figures 5.2(d)-(e), completing the proof of the claim.

To conclude the proof, we need to show that one can take $\bar{t}=T$. Let $\bar{t} \in[0, T]$ be the maximal time such that $\{x(t): t \in[0, \bar{t}]\}$ is the union of extremal arcs. Suppose that $\bar{t} \in(0, T)$, and set $x_{1}=x(\bar{t})$. Since, by assumption, $\Sigma$ is not STLC from $x(t)$ for all $t \in[0, T]$, application of the claim at $x_{1}$ implies that there exists $t_{1}>\bar{t}$ such that $\left\{x(t): t \in\left[\bar{t}, t_{1}\right]\right\}$ is the union of extremal arcs. Hence, $\left\{x(t): t \in\left[0, t_{1}\right]\right\}$ is the union of extremal arcs, contradicting the maximality of $\bar{t}$ and proving that $\bar{t}=T$.

The boundary of invariance kernels enjoys the so-called barrier property.

Proposition 5.2 (barrier property [23]). Let $K^{\star}$ be the invariance kernel of $K$ for (1.1), and assume it is not empty. Then for any $x_{0}$ in $\partial K^{\star}$ there exists $\lambda(t) \in \mathcal{U}$ such that the solution to (1.1) with initial condition $x(0)=x_{0}$ remains in $\partial K^{\star}$ for all $t \geq 0$, or until it reaches $\partial K$.

We stress that the proof of this result is completely analogous to the proof of Theorem 4.18 in [4].

Proof. Since $K^{\star}$ is the invariance kernel of $K$, for any point $x_{0}$ in $K \backslash K^{\star}$ there exist $\lambda(t) \in \mathcal{U}$ and $\bar{t}>0$ such that the solution of (1.1) with initial condition $x_{0}$ does not enter $K^{\star}$ and remains in $K$ for all $t \in[0, \bar{t}]$. The construction that follows is illustrated in Figure 5.3. Let $\bar{x}$ be an arbitrary point of $\partial K^{\star} \backslash \partial K$ and take a sequence $\left\{x_{i}\right\} \subset K \backslash K^{\star}$ with $x_{i} \rightarrow \bar{x}$. For each $i \in \mathbb{N}$, there exist $\lambda_{i}(t) \in \mathcal{U}$ and $T_{i}>0$ such that the solution $x_{i}(t)$ of (1.1) with initial condition $x(0)=x_{i}$ remains in $K \backslash K^{\star}$ for all $t \in\left[0, T_{i}\right]$. Moreover, the fact that $\bar{x} \notin \partial K$ implies that $T=\inf _{i} T_{i}$ is positive. Now consider the sequence of functions $x_{i}(t), t \in[0, T]$. Since $x_{i}(t)$ are trajectories of the locally Lipschitz (and hence upper semicontinuous) differential inclusion (1.2), and since $x_{i}(0) \rightarrow \bar{x}$, the sequence $\left\{x_{i}(t)\right\}$ converges uniformly to a function $\bar{x}(t), t \in[0, T]$ which is a trajectory of (1.2) through the initial condition $\bar{x} \in \partial K^{\star}$. Equivalently, there exists $\lambda(t) \in \mathcal{U}$ such that $\bar{x}(t)$ is the solution of (1.1) with initial condition $\bar{x}$. 
Since $\bar{x} \in \partial K^{\star}$, and since the invariance kernel $K^{\star}$ is a closed set, we have $\bar{x}(t) \in K^{\star}$ for all $t \in[0, T]$. At the same time, since $\bar{x}(t)$ is the uniform limit of the sequence $\left\{x_{i}(t)\right\}$ and $x_{i}(t) \in K \backslash K^{\star}$ for all $t \in[0, T]$, we have that $\bar{x}(t) \in \overline{K \backslash K^{\star}}$. In conclusion $\bar{x}(t) \in K^{\star} \cap \overline{K \backslash K^{\star}}=\partial K^{\star}$ for all $t \in[0, T]$. We have thus shown that for all $\bar{x} \in \partial K^{\star} \backslash \partial K$ there exists a time $T>0$ and $\lambda(t) \in \mathcal{U}$ such that the solution of $\Sigma$ with initial condition $\bar{x}$ remains in $\partial K^{\star}$ for all $t \in[0, T]$. This fact readily implies the statement of the theorem.

Lemma 5.1 and Proposition 5.2 yield the following.

Proposition 5.3. If $K^{\star}$ is nonempty, then each connected component of $\partial K^{\star}$ is a concatenation including extremal arcs and invariant arcs of $\partial K$.

Proof. Let $x_{0}$ be an arbitrary point in $\partial K^{\star} \backslash \partial K$. Since $K^{\star}$ is closed and positively invariant, for all $T>0$ we have $\mathcal{A}\left(x_{0},[0, T]\right) \subset K^{\star}$ which, by the fact that $x_{0} \in \partial K^{\star}$, implies that $x_{0} \in \partial \mathcal{A}\left(x_{0},[0, T]\right)$ for all $T>0$. Thus, $\Sigma$ is not STLC from $x_{0}$ and, since $x_{0}$ is arbitrary, $\Sigma$ is not STLC from any point on $\partial K^{\star} \backslash \partial K$. By Proposition 5.2, there exists $\lambda(t) \in \mathcal{U}$ and $T>0$ such that the corresponding solution $\bar{x}(t)$ of $\Sigma$ through $x_{0}$ remains in $\partial K^{\star}$ for all $t \in[0, T]$. We thus have that

$$
(\forall t \in[0, T]) \bar{x}(t) \in \mathcal{A}\left(x_{0}, t\right) \cap \partial K^{\star}=\partial \mathcal{A}\left(x_{0}, t\right) \cap \partial K^{\star},
$$

where the equality in the above relation is due to the fact that $\mathcal{A}\left(x_{0}, t\right) \subset K^{\star}$ for all $t \in[0, T]$. In particular, then, $\bar{x}(t) \in \partial \mathcal{A}\left(x_{0}, t\right)$ for all $t \in[0, T]$ and $\Sigma$ is not STLC from $\bar{x}(t)$ for all $t \in[0, T]$. By Lemma $5.1, \bar{x}(t)$ is a concatenation of extremal solutions. On the other hand, for any $x_{0} \in \partial K^{\star} \cap \partial K$, all solutions of $\Sigma$ through $x_{0}$ must remain in $K$ for all positive time which, by Theorem 4.3 .8 in [12], implies that $f_{1}\left(x_{0}\right)$ and $f_{2}\left(x_{0}\right)$ must point inside of $K$ or be tangent to $\partial K$ at $x_{0}$. Therefore, the set $\partial K^{\star} \cap \partial K$ is the union of invariant $\operatorname{arcs}$ of $\partial K$.

The proof of Lemma 5.1 and the barrier property in Proposition 5.2 give the following.

Lemma 5.4. The only equilibria of $f_{1}$ and $f_{2}$ that may belong to $\partial K^{\star}$ are nodes (stable or unstable) and saddle points, and the only points in $\partial K^{\star} \cap \mathcal{L}^{-}$are $t^{-}$points.

Proof. By assumption (iii) in section 3, all equilibria are hyperbolic, so they are either foci, saddles, or nodes. Let $\bar{x} \in \partial K^{\star}$ be either a focus or a point in $\mathcal{L}^{-}$which is not a $t^{-}$point. As argued in the proof of Lemma 5.1 (Cases 3 and 4 ), $\Sigma$ is STLC from $\bar{x}$. Therefore, for all $T>0, \mathcal{A}(\bar{x},[0, T])$ contains $\bar{x}$ in its interior, implying that $\mathcal{A}(\bar{x},[0, T])$ is not entirely contained in $K^{\star}$. Since $\bar{x} \in \partial K^{\star}$ and $K^{\star}$ is a closed set, the above contradicts the positive invariance of $K^{\star}$.

6. Concatenation of extremal arcs and invariant arcs of $\partial K$. Proposition 5.3 indicates that the boundary of the invariance kernel $K^{\star}$ is formed by concatenations of extremal arcs and invariant segments of $\partial K$. The result below identifies all feasible concatenations on $\partial K^{\star}$. Before stating the proposition, we introduce some notation. We will use the shorthands HH, HT, TT to signify "head-to-head," "headto-tail," and "tail-to-tail," respectively. The notation $\mathrm{A} \rightarrow \stackrel{p}{\leftarrow} \leftarrow \mathrm{B}$ will be used to indicate an $\mathrm{HH}$ concatenation at point $p$ between $\operatorname{arcs} \mathrm{A}$ and $\mathrm{B}$, where the symbols $\mathrm{A}, \mathrm{B}$ belong to the list $\{\mathrm{L}, \mathrm{R}, \partial K\}$. ( $\partial K$ stands for invariant $\operatorname{arc}$ of $\partial K$.) Similarly, $\mathrm{A} \rightarrow \stackrel{p}{\rightarrow} \mathrm{B}$, $\mathrm{A} \stackrel{p}{\longrightarrow} \mathrm{B}$ will be used to indicate HT and TT concatenations, respectively. ${ }^{2}$ To state that a concatenation occurs at a saddle or node (stable or unstable) of $f_{1}$ or $f_{2}$ (recall that foci are ruled out by Lemma 5.4) we will set $p=0$, while to state that the concate-

\footnotetext{
${ }^{2}$ Since the tail-to-head concatenation of two $\operatorname{arcs} \mathrm{A}$ and B is the head-to-tail concatenation of B and $\mathrm{A}$, we do not need to introduce notation for tail-to-head concatenations.
} 
nation occurs anywhere on a set $S$ we will set $p=S$. If $p$ is omitted, then the location of the concatenation is unspecified. To illustrate, $\mathrm{L} \stackrel{t^{\partial}}{\rightarrow} \partial K$ denotes an HT concatenation of an L-arc with an invariant arc of $\partial K$ at a $t^{\partial}$ point, and $\partial K \stackrel{\partial K}{\rightarrow} \mathrm{L}$ denotes an HT concatenation of an invariant arc of $\partial K$ and an L-arc occurring anywhere on $\partial K$.

Proposition 6.1. On $\partial K^{\star}$, the only feasible concatenations involving extremal arcs and invariant arcs of $\partial K$ are

(HH) $L \stackrel{\circ}{\leftrightarrow} \leftarrow R, L \stackrel{t^{-}}{\leftarrow} \leftarrow R, \partial K \stackrel{t^{\partial}}{\leftarrow} \leftarrow R$,

(HT) $L \stackrel{t^{\partial}}{\rightarrow} \partial K, \partial K \stackrel{\partial K}{\rightarrow} L, L \rightarrow \rightarrow L, R \rightarrow \rightarrow R$,

(TT) $\partial K \stackrel{\partial K}{\longrightarrow} R, L \stackrel{\bar{x}}{\rightarrow} R$, where $\bar{x}$ is either a $t^{-}$point or any point in $\left(\mathcal{L}^{-}\right)^{c}$.

Proof. By way of contradiction, assume that $\partial K^{\star}$ contains arc concatenations that are not of the type listed in the proposition statement. The following nine arguments enumerate the possible cases left out by the statement, grouping them by concatenation type: HH (Cases 1-4), HT (Case 5), and TT (Cases 6-9). Within each concatenation type, the arguments address the types of points and arcs left out by the proposition statement.

1. $\mathrm{L} \rightarrow \stackrel{\bar{x}}{\leftarrow} \mathrm{R}$, where $\bar{x}$ is not an equilibrium, $t^{-}$, or $t^{\partial}$ point. We distinguish two cases: $\bar{x} \in \partial K^{\star} \cap \partial K$ and $\bar{x} \in \partial K^{\star} \backslash \partial K$. If $\bar{x} \in \partial K^{\star} \cap \partial K$, in order for the Land R-arcs to meet $\mathrm{HH}$ at $\bar{x}$ it is necessary that they are both tangent to $\partial K$ at $\bar{x}$, for otherwise either $f_{1}(\bar{x})$ or $f_{2}(\bar{x})$ would point outside of $K$ at $\bar{x}$. Since both L- and R-arcs are tangent to $\partial K$ at $\bar{x}$ and they meet $\mathrm{HH}$ at $\bar{x}, f_{1}(\bar{x})$ and $f_{2}(\bar{x})$ are antiparallel at $\bar{x}$, i.e., $\bar{x} \in \partial K^{\star} \cap \mathcal{L}^{-}$. By Lemma 5.4, $\bar{x}$ must be a $t^{-}$point, contradicting the assumption of Case 1. If $\bar{x} \in \partial K^{\star} \backslash \partial K$, then the L- and R-arcs through $\bar{x}$ cannot both enter the interior of $K^{\star}$, for otherwise the only way that the barrier property could hold at $\bar{x}$ is if $\bar{x}$ is a constant solution of $\Sigma$, and this can only happen if $0 \in \operatorname{co}\left\{f_{1}(\bar{x}), f_{2}(\bar{x})\right\}$, i.e., $\bar{x} \in \partial K^{\star} \cap \mathcal{L}^{-}$. By Lemma $5.4, \bar{x}$ must be a $t^{-}$ point, contradicting the assumption of Case 1 . It must therefore be the case that at least one of the L- and R-arcs through $\bar{x}$, say, the L-arc, remains in $\partial K^{\star}$ for some positive time. Let $A$ and $B$ denote, respectively, the L- and R-arcs in $\partial K^{\star}$ that meet $\mathrm{HH}$ at $\bar{x}$, and let $C$ denote the L-arc through $\bar{x}$ that remains in $\partial K^{\star}$ for some positive time. The situation just described can only occur if $B \cap C$ is an extremal arc of positive measure containing $\bar{x}$, implying that $f_{1}$ and $f_{2}$ are antiparallel at all points in $B \cap C$, i.e., $B \cap C \subset \mathcal{L}^{-}$and all points in $B \cap C$ are $t^{-}$points. This situation is ruled out by assumption (ii) in section 3 .

2. $\mathrm{L} \stackrel{t^{\partial}}{\leftarrow} \leftarrow \mathrm{R}$. By assumption (vi), none of the $t^{\partial}$ points lies in $\mathcal{L}$, and therefore the L- and R-arcs meet $\mathrm{HH}$ at a $t^{\partial}$ point in $\partial K$ and are not tangent to each other. It follows that at the concatenation point either $f_{1}$ or $f_{2}$ points outside of $K$, which contradicts the definition of $t^{\partial}$ point.

3. $\mathrm{L} \rightarrow \leftarrow \mathrm{L}, \mathrm{R} \rightarrow \leftarrow \mathrm{R}$, or $\mathrm{L} \rightarrow \leftarrow \partial K$. In all these cases, the concatenation would produce an arc which is not consistently crossed on one side by $\operatorname{arcs}$ of $\Sigma$, violating the positive invariance of $K^{\star}$.

4. $\partial K \rightarrow \stackrel{\bar{x}}{\leftarrow} \mathrm{R}$, where $\bar{x} \in \partial K$ is not a $t^{\partial}$ point. If an R-arc in $\partial K^{\star}$ meets HH with an invariant arc of $\partial K$ at a point $\bar{x}$, then since the $\mathrm{R}$-arc through $\bar{x}$ remains in $K^{\star}$ in positive time, it must be the case that the R-arc is tangent to $\partial K$ at $\bar{x}$, which implies that either $f_{1}$ or $f_{2}$ is tangent to $\partial K$ at $\bar{x}$. By assumption (ii), both $f_{1}$ and $f_{2}$ are not tangent to $\partial K$ in a punctured neighborhood of $\bar{x}$. By assumption (iv), $\bar{x}$ is not an equilibrium of $f_{1}$ and $f_{2}$. The situation resulting from these conditions, depicted in Figure 6.1(a), is compatible only with the case when $\bar{x}$ is a $t^{\partial}$ point. This is because in any neighborhood of $\bar{x}$ there are points in $\partial K$ at which $f_{1}$ or $f_{2}$ points outside of $K$. 


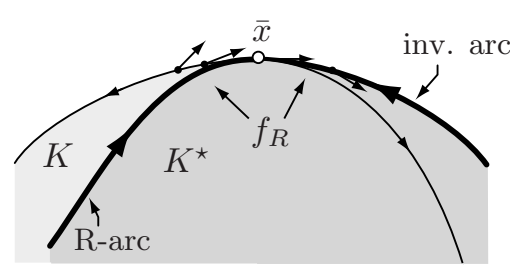

(a)

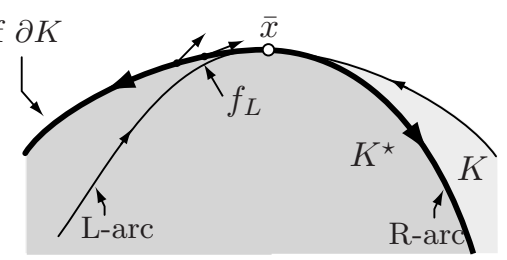

(b)

FIG. 6.1. Illustration of arguments in the proof of Proposition 6.1.

5. $\mathrm{R} \rightarrow \rightarrow \partial K, \partial K \rightarrow \rightarrow \mathrm{R}, \mathrm{R} \rightarrow \rightarrow \mathrm{L}, \mathrm{L} \rightarrow \rightarrow \mathrm{R}$. In all these cases, the resulting concatenation would produce an arc which is not consistently crossed on one side by $\operatorname{arcs}$ of $\Sigma$.

6. $\mathrm{L} \stackrel{\bar{x}}{\leftrightarrow} \mathrm{R}$, where $\bar{x} \in \mathcal{L}^{-}$is not a $t^{-}$point. By Lemma 4.1 , if $\bar{x} \in \mathcal{L}^{-}$is not a $t^{-}$ point, then L- and R-arcs can only meet HT at $\bar{x}$, and therefore this concatenation cannot occur.

7. $\partial K \stackrel{\bar{x}}{\rightarrow} \mathrm{R}$, where $\bar{x} \in \mathcal{L}^{-}$is not a $t^{-}$point. Since the vectors $f_{1}(\bar{x})$ and $f_{2}(\bar{x})$ are antiparallel, in order for $\bar{x}$ to belong to $\partial K^{\star}$ it is necessary that both $f_{1}(\bar{x})$ and $f_{2}(\bar{x})$ are tangent to $\partial K$. Moreover, by Lemma 4.1 each L-arc through $\bar{x}$ meets the invariant arc of $\partial K \mathrm{HT}$ at $\bar{x}$. This situation, depicted in Figure 6.1(b), violates the invariance of the $\partial K$ arc.

8. $\mathrm{L} \leftarrow \rightarrow \mathrm{L}, \mathrm{R} \leftarrow \rightarrow \mathrm{R}, \partial K \leftarrow \rightarrow \partial K$. None of the arc pairs above can meet TT, and even if they could, the resulting arc would not be consistently crossed on one side by $\operatorname{arcs}$ of $\Sigma$.

9. $\partial K \leftarrow \rightarrow \mathrm{L}$. This concatenation is not consistently crossed on one side by arcs of $\Sigma$.

7. Main result. In this section we present the main theoretical result of this paper characterizing the boundary of the invariance kernel. This result relies on Proposition 6.1 and other properties proved earlier.

THEOREM 7.1. Each connected component of $\partial K^{\star}$ is either a closed extremal arc, a closed invariant arc of $\partial K$, or the concatenation of extremal arcs and invariant arcs of $\partial K^{\star}$ according to the rules listed in Proposition 6.1. An extremal arc which is not closed can only be part of $\partial K^{\star}$ if one of its endpoints is a $t^{\partial}$ point, a $t^{-}$point, or an equilibrium (saddle or node) of $f_{1}$ or $f_{2} . \gamma$ is a permissible equilibrium extremal arc through a node on $\partial K^{\star}$ only if at least one of the following holds:

(i) $\gamma$ coincides with the fast manifold of $\bar{x}$ locally around $\bar{x}$.

(ii) $\gamma$ is the nonequilibrium extremal arc of another equilibrium (saddle or node of $f_{1}$ or $f_{2}$ ) or an extremal arc through a $t^{-}$or $t^{\partial}$ point.

(iii) $\gamma$ is simultaneously an equilibrium extremal arc for $\bar{x}$ and for another equilibrium $\bar{y} \neq \bar{x}$. In this case, either $\gamma$ is of type (i), or locally around $\bar{y}, \gamma$ coincides with the stable/unstable manifold of $\bar{y}$, if $\bar{y}$ is a saddle, or the fast manifold of $\bar{y}$ if $\bar{y}$ is a node.

Proof. Suppose, by way of contradiction, that $\partial K^{\star}$ contains an extremal arc $\gamma$ which is not closed and whose endpoints violate the conditions of the theorem. By Proposition 6.1, the head of an extremal arc can only be concatenated at an equilibrium of $f_{1}$ or $f_{2}$, or at $t^{-}, t^{\partial}$ points. By the contradiction assumption, we exclude concatenations at $t^{-}, t^{\partial}$ points. The only possibility left is that the the head of $\gamma$ must be a node $\bar{x}$, and $\gamma$ must be an equilibrium extremal arc which does not belong to any of the types (i)-(iii) in the theorem statement. Suppose, without loss of 


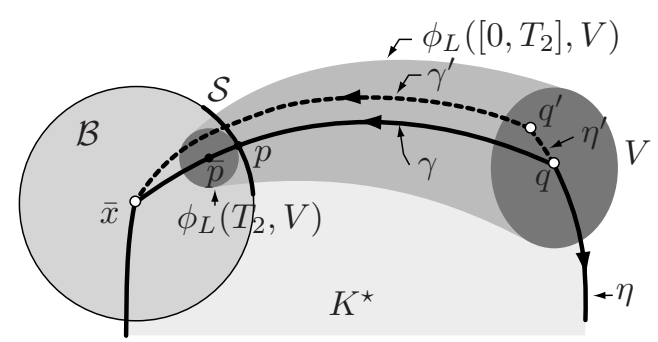

(a)

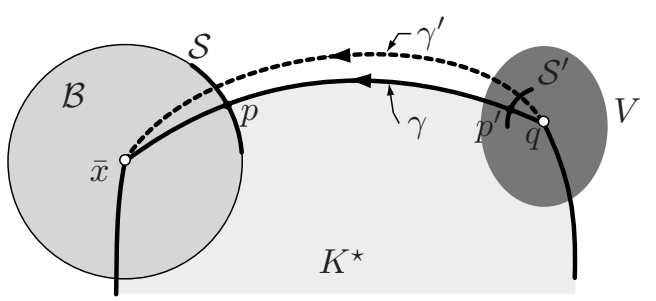

(b)

FIG. 7.1. Illustration of proof of Theorem 7.1.

generality, that $\gamma$ is an L-arc. Some of the constructions that follow are illustrated in Figure 7.1. Since in any neighborhood of $\bar{x} \gamma$ does not coincide with the fast manifold of $\bar{x}$, by Lemma 4.3 there exists a ball $\mathcal{B}$ centered at $\bar{x}$ and a circle segment $\mathcal{S} \subset \partial \mathcal{B}$ with a unique intersection point $p=\mathcal{S} \cap \gamma$ such that all L-arcs through points in $\mathcal{S}$ remain in $\mathcal{B}$ in positive time and are all equilibrium extremal arcs. Since $\bar{x} \in \partial K^{\star} \backslash \partial K$, the ball $\mathcal{B}$ can be taken small enough that $p \in \partial K^{\star} \backslash \partial K$ as well. Let $\bar{p} \in \gamma$ be a point in the interior of $\mathcal{B}$ and denote by $q$ the tail of $\gamma$. Then, there exist $T_{2}>T_{1}>0$ such that $\phi_{L}\left(T_{1}, q\right)=p$ and $\phi_{L}\left(T_{2}, q\right)=\bar{p}$. By the contradiction assumption, $\gamma$ does not contain $t^{-}$points and so by Lemma 5.4 it follows that $\gamma \cap \mathcal{L}^{-}=\varnothing$. Consequently, by Lemma 4.2 there exists a neighborhood $U$ of $q$ such that the map $\phi_{L}:\left[0, T_{2}\right] \times U \rightarrow \mathbb{R}^{2}$ is continuous. By continuity, there exists a neighborhood $V \subset U$ of $q$ such that the following two properties hold:

(a) $\phi_{L}\left(\left[0, T_{2}\right], V\right) \cap \partial \mathcal{B} \subset \mathcal{S}$,

(b) $\phi_{L}\left(T_{2}, V\right) \subset \mathcal{B}$.

The two properties above imply that all L-arcs through points in $V$ intersect $S$ and, by Lemma 4.3, they are equilibrium extremal arcs, i.e., their head is at $\bar{x}$. Next, we investigate the available concatenations at the tail $q$ of $\gamma$. According to Proposition 6.1, the only possible tail concatenations of $\gamma$ at $q$ are $\partial K^{\partial K} \rightarrow \gamma$ and $\gamma \stackrel{q}{\rightarrow} \mathrm{R}$. For the latter concatenation, $q$ must be either a $t^{-}$point, or any point in $\left(\mathcal{L}^{-}\right)^{c}$. Our contradiction assumption rules out $t^{-}$points, so $q$ must be either a point on $\partial K$ or a point in $\left(\mathcal{L}^{-}\right)^{c}$. A special case of interest is when $q$ is in $\left(\mathcal{L}^{-}\right)^{c}$ and $q$ is an equilibrium of $f_{1}$ or $f_{2}$. In this case, the contradiction assumption implies that $q$ must be a node and that $\gamma$ must be an equilibrium extremal arc through $q$ which, near $q$, does not coincide with the fast manifold of $q$. Summarizing our observations so far, there are three possibilities for the tail $q$ of $\gamma$ : (1) $q \in\left(\mathcal{L}^{-}\right)^{c} \backslash \partial K$, and $q$ is not an equilibrium; (2) $q \in \partial K$; (3) $q \in\left(\mathcal{L}^{-}\right)^{c} \backslash \partial K$ is a node, and $\gamma$ is an equilibrium extremal arc through $q$ which, near $q$, does not coincide with the fast manifold of $q$. We now investigate these three cases in detail.

Case 1. $q \in\left(\mathcal{L}^{-}\right)^{c} \backslash \partial K$ is not an equilibrium. Since by the contradiction assumption, $\gamma$ does not contain $t^{-}$and $t^{\partial}$ points, we also have that $\gamma \in\left(\mathcal{L}^{-}\right)^{c} \backslash \partial K$. Moreover, we can assume that $V$ is small enough that $V \subset \operatorname{int} K$. By Proposition 6.1, at $q$ there must be a TT concatenation between $\gamma$ and an R-arc $\eta$. Extend $\eta$ in negative time from $q$ and denote by $\eta^{\prime}$ the extended arc. If $q \notin \mathcal{L}^{+}$, then $f_{1}(q)$ and $f_{2}(q)$ are linearly independent. Therefore, in a neighborhood of $q$, without loss of generality $V$, the arc $\eta^{\prime}$ is transversal to all L-arcs. If, on the other hand, $q \in \mathcal{L}^{+}$, then by assumption (vii) in section $3, \eta^{\prime}$ is transversal to L-arcs in a punctured neighborhood of $q$, without loss of generality in $V \backslash\{q\}$. In both cases, in any neighborhood of $q$ contained in $V$ 
there exists $q^{\prime} \in \eta^{\prime} \cap V$ with the property that $q^{\prime} \notin K^{\star}$, and therefore such that the L-arc $\gamma^{\prime}$ through $q^{\prime}$ is not contained in $K^{\star}$. Since $q^{\prime} \in V, \gamma^{\prime}$ has its head at $\bar{x}$. Since $\gamma \subset\left(\mathcal{L}^{-}\right)^{c} \backslash \partial K$, an open set, $q^{\prime}$ can be chosen such that $\gamma^{\prime} \subset\left(\mathcal{L}^{-}\right)^{c} \backslash \partial K$ as well. The set obtained from $K^{\star}$ by replacing the concatenation $\varkappa^{q} \rightarrow \eta$ with $\gamma^{\prime} \stackrel{q^{\prime}}{\rightarrow} \rightarrow \eta^{\prime}$ is contained in $K$, is positively invariant, and contains $K^{\star}$, contradicting the assumption that $K^{\star}$ is the invariance kernel of $K$. This construction is illustrated in Figure 7.1(a).

Case 2. $q \in \partial K$. Since by the contradiction assumption $q$ is not a $t^{\partial}$ point, it follows that $q$ is not the endpoint of an invariant $\operatorname{arc}$ of $\partial K$. If the vectors $f_{1}(q), f_{2}(q)$ point to the interior of $K$, then the invariant arc of $\partial K$ containing $q$ is transversal to L-arcs in a neighborhood of $q$, without loss of generality in $V$. If, on the other hand, $f_{1}(q)$ or $f_{2}(q)$ is tangent to $\partial K$, then by assumption (ii) in section 3 the invariant arc of $\partial K$ containing $q$ is transversal to L-arcs in a punctured neighborhood of $q$, without loss of generality in $V \backslash\{q\}$. In both cases, in any neighborhood of $q$ contained in $V$ there exists $q^{\prime} \in \partial K$ such that the L-arc $\gamma^{\prime}$ through $q^{\prime}$ is contained in $K$ but is not contained in $K^{\star}$ and has head at $\bar{x}$. As before, replacing the concatenation $\gamma \stackrel{q}{\rightarrow} \eta \eta$ with $\gamma^{\prime} \stackrel{q^{\prime}}{\longleftarrow} \rightarrow \partial K$ we obtain a positively invariant set contained in $K$ which contains $K^{\star}$, a contradiction.

Case 3. $q \in\left(\mathcal{L}^{-}\right)^{c} \backslash \partial K$ is a node and $\gamma$ is an equilibrium extremal arc through $q$ which, near $q$, does not coincide with the fast manifold of $q$. By Lemma 4.3, there exists a ball $\mathcal{B}^{\prime}$ centered at $q$ and a circle segment $\mathcal{S}^{\prime} \subset \partial \mathcal{B}^{\prime}$ with a unique intersection point $p^{\prime}=\mathcal{S}^{\prime} \cap \gamma$ such that all L-arcs through $\mathcal{S}^{\prime}$ remain in $\mathcal{B}^{\prime}$ in negative time and are equilibrium extremal arcs through $q$. We can assume that $\mathcal{S}^{\prime} \subset V$. (If that isn't the case, we can make $\mathcal{B}^{\prime}$ smaller.) Thus, all L-arcs through $S^{\prime}$ have tail at $q$ and head at $\bar{x}$. In particular, one can choose a point on $\mathcal{S}^{\prime}$ outside of $\mathcal{K}^{\star}$ through which there is an L-arc $\gamma^{\prime}$ with tail at $q$ and head at $\bar{x}$ such that $\gamma^{\prime} \not \subset K^{\star}$ but $\gamma^{\prime} \subset K$. By replacing $\gamma$ with $\gamma^{\prime}$ we enlarge $K^{\star}$ and get a contradiction.

Remark 3. The significance of the theorem above is that in conjunction with Proposition 6.1, it identifies a finite number of extremal arcs that may be part of $\partial K^{\star}$, thus making it possible to develop an algorithm to construct all permissible boundaries of the invariance kernel $K^{\star}$. The arcs in question have head or tail at $t^{-}$points, $t^{\partial}$ points, nodes, or saddles. By Lemma 4.1, L- and R-arcs through $t^{-}$and $t^{\partial}$ points are unique. Through each node or saddle, there are two nonequilibrium extremal arcs and multiple equilibrium extremal arcs as follows. There are four equilibrium extremal arcs through a saddle, coinciding in a neighborhood of the saddle with the stable and unstable manifolds, and there are infinitely many equilibrium extremal arcs through a node. However, the theorem states that only finitely many such arcs are admissible, specifically the ones of types (i)-(iii).

8. Invariance kernel algorithm. In the exposition of this algorithm, it is assumed that any closed extremal arcs are known. Moreover, it is assumed that $K$ is not positively invariant, for in this case trivially $K^{\star}=K$. The problem of theoretically and practically establishing the existence of a closed extremal trajectory is similar to doing so for the closed periodic orbits of a dynamical system. A rigorous proof of existence could be based on Poincaré return maps. An engineering approach of examining the streamlines of the $f_{L}$ and $f_{R}$ fields would likely be sufficient to detect and compute such trajectories in order to apply this algorithm.

The algorithm has five parts. In the initialization part, one identifies points of interest. In the integration part, one generates suitable extremal arcs through the special points. In the pruning part, one eliminates portions of extremal arcs that are not relevant for the construction of $\partial K^{\star}$. In the graph construction part, one 
TABLE 8.1

Rules of integration through the special points identified in part 1 of the algorithm. For any such point, this table specifies which extremal field to integrate and whether the integration is in forward or reverse time. Note that at a node one generates four arcs. Two are the nonequilibrium extremal arcs originating and ending at the node, while the other two are those extremal arcs that, in a neighborhood of the node, coincide with its fast manifold. Similarly, at a saddle point we generate the nonequilibrium extremal arcs and those equilibrium arcs that locally coincide with its stable and unstable manifolds.

\begin{tabular}{|c|c|c|c|}
\hline \multicolumn{2}{|c|}{$\begin{array}{c}\text { Initial } \\
\text { condition }\end{array}$} & $\begin{array}{c}\text { Extremal } \\
\text { arc }\end{array}$ & $\begin{array}{c}\text { Integration } \\
\text { direction }\end{array}$ \\
\hline \multirow{3}{*}{$\begin{array}{l}t^{\partial} \text { point, } \\
\text { tail of inv. arc }\end{array}$} & \multirow{2}{*}{$\begin{array}{c}f_{L} \text { is } \\
\text { tangent }\end{array}$} & $\mathrm{L}$ & rev. \\
\hline & & $\mathrm{R}$ & fwd. \\
\hline & $\begin{array}{c}f_{R} \text { is } \\
\text { tangent }\end{array}$ & \multicolumn{2}{|c|}{ do nothing } \\
\hline \multirow{3}{*}{$\begin{array}{c}t^{\partial} \text { point, } \\
\text { head of inv. arc }\end{array}$} & $\begin{array}{c}f_{L} \text { is } \\
\text { tangent }\end{array}$ & \multicolumn{2}{|c|}{ do nothing } \\
\hline & \multirow{2}{*}{$\begin{array}{c}f_{R} \text { is } \\
\text { tangent }\end{array}$} & $\mathrm{L}$ & fwd. \\
\hline & & $\mathrm{R}$ & rev. \\
\hline \multirow{4}{*}{\multicolumn{2}{|c|}{$t^{-}$point }} & $\mathrm{L}$ & fwd. \\
\hline & & $\mathrm{L}$ & rev. \\
\hline & & $\mathrm{R}$ & fwd. \\
\hline & & $\mathrm{R}$ & rev. \\
\hline \multirow{4}{*}{\multicolumn{2}{|c|}{$\begin{array}{c}\text { node } \\
\text { stable or (unstable) }\end{array}$}} & \multirow[t]{2}{*}{ noneq. } & fwd. \\
\hline & & & rev. \\
\hline & & \multirow{2}{*}{$\begin{array}{l}\text { eq., fast } \\
\text { manifold }\end{array}$} & rev. (fwd.) \\
\hline & & & rev. (fwd.) \\
\hline \multirow{6}{*}{\multicolumn{2}{|c|}{ saddle }} & \multirow[t]{2}{*}{ noneq. } & fwd. \\
\hline & & & rev. \\
\hline & & \multirow{2}{*}{$\begin{array}{l}\text { eq., stable } \\
\text { manifold }\end{array}$} & rev. \\
\hline & & & rev. \\
\hline & & \multirow{2}{*}{$\begin{array}{l}\text { eq., unstable } \\
\text { manifold }\end{array}$} & fwd. \\
\hline & & & fwd. \\
\hline
\end{tabular}

associates to the collection of arcs a graph which is used to find invariant boundaries. Finally, the simple cycles of the graph are used to determine $K^{\star}$.

\section{Initialization}

Determine:

1.1. $t^{\partial}$ points in $K$,

1.2. $t^{-}$points in $K$,

1.3. nodes and saddles of $f_{1}$ or $f_{2}$ in $K$,

1.4. closed extremal arcs in $K$.

\section{Integration}

Using the integration rules in Table 8.1, generate extremal arcs from all points computed in part 1. The stopping criteria for the integration are:

2.1. The solution hits $\mathcal{L}^{-}$at a point which is not a $t^{-}$point.

2.2. The solution hits $\partial K$ at a point which does not lie on an invariant arc of $\partial K$.

2.3. The solution hits an invariant arc of $\partial K$ coming from int $K$.

2.4. The solution is detected to reach (in finite or infinite time) an equilibrium of $f_{1}$ or $f_{2}$ or to spiral (in positive or negative time) around a limit set.

\section{Pruning}

Label all points identified in part 1 (steps 1.1-1.4) as special points. Label as significant all special points, all the integration endpoints, and all points of intersection between extremal arcs generated in part 2 or between extremal and invariant $\operatorname{arcs}$ of $\partial K$. Thus, special points are significant, but not vice versa. 
3.1. Partition each extremal arc resulting from an integration performed in part 2 and invariant arcs of $\partial K$ into subarcs whose heads and tails are the significant points. The subarcs inherit the orientation of the parent arc. In the rest of the algorithm below, these subarcs will be simply referred to as extremal arcs.

3.2. Prune one L-arc $\gamma$ and one R-arc $\eta$ if $\gamma$ and $\eta$ have the same endpoints, and if neither endpoint is special.

3.3. Prune any L-arc (resp., R-arc) with head at a point $p$ which is not special if there is no L-arc (resp., $\mathrm{R}$-arc) with tail at $p$.

3.4. Prune any extremal arc whose head or tail is at a point where no other arc is connected.

3.5. Repeat steps 3.3-3.4 until there is no more arc to prune.

3.6. Prune extremal arcs that spiral around limit sets in positive or negative time.

3.7. Eliminate from the list of significant points all points with no arcs attached, and points connecting only two arcs of the same type (L or R).

4. Graph construction

Construct a graph $\mathcal{G}=(\mathcal{V}, \mathcal{E})$, with $\mathcal{V}$ the set of vertices of $\mathcal{G}$ and $\mathcal{E}$ the set of edges of $\mathcal{G}$ as follows.

Vertices of $\mathcal{G}$. Let $\mathcal{P}$ denote the set of significant points in $K$ that remain after the pruning in part 3.

4.1. For every point $p \in \mathcal{P}$ which is special, create a vertex $v_{p}$.

4.2. For every $p \in \mathcal{P}$ which is not special, create two vertices, denoted $v_{p}^{L}$ and $v_{p}^{R}$.

Edges of $\mathcal{G}$. Create directed edges between vertices associated with extremal arcs and invariant arcs of $\partial K$ as follows:

4.3. If $p$ is the tail of an L-arc or an invariant arc of $\partial K$ with head at $q$, create a directed edge from $v_{p}$, or $v_{p}^{L}$, to $v_{q}$, or $v_{q}^{L}$.

4.4. If $p$ is the tail of an R-arc with head at $q$, create a directed edge from $v_{q}$, or $v_{q}^{R}$, to $v_{p}$, or $v_{p}^{R}$.

4.5. For every $\left(v_{p}^{L}, v_{p}^{R}\right)$ pair, create a directed edge from $v_{p}^{R}$ to $v_{p}^{L}$.

\section{Cycle analysis}

5.1. Find all simple cycles (i.e., closed paths that do not visit any vertex more than once) in the graph $\mathcal{G}$.

5.2. Discard any cycles containing two vertices $v_{p}^{R}, v_{p}^{L}$ that are not consecutive (when travelling in the direction of the edges of the graph).

5.3. For each remaining cycle in $\mathcal{G}$, check whether the region in the plane delimited by the path associated with the cycle is positively or negatively invariant. If it is negatively invariant, discard the cycle.

5.4. $K^{\star}$ is the union of all regions enclosed by closed paths associated with graph cycles and by closed extremal trajectories in $K$.

Remark 4 . The simple cycles of $\mathcal{G}$ can be efficiently found using Tarjan's algorithm in [28], which has polynomial complexity $O((V \cdot E)(C+1))$, where $V, E, C$ are the number of vertices, edges, and simple cycles in $\mathcal{G}$.

The test in step 5.3 can be done simply by picking a point $p$ in the closed path that is not a special point and discarding the cycle if $f_{1}(p)$ points outside the region delimited by the path.

Remark 5. As shown in the next section, the proposed algorithm determines the invariance kernel exactly in a finite number of steps. In practice, the only source of approximation error is the integration of extremal vector fields to determine the 
extremal arcs in step 2 of the algorithm. To perform this integration, one can use any numerical ODE solver. In so doing, one can set the solver's tolerances so as to obtain an arbitrarily small approximation error in the determination of $K^{\star}$. More precisely, for any $\epsilon>0$ one can choose the integration tolerances to ensure that the Hausdorff distance between the actual $K^{\star}$ and its approximation is less than $\epsilon$. From this observation it immediately follows that if the invariance kernel is stable under perturbations of the problem data (i.e., if small perturbations of $f_{1}, f_{2}$, or $K$ give rise to a small perturbation of $K^{\star}$ ), then our algorithm is also stable with respect to these perturbations.

9. Algorithm justification. The algorithm presented in the previous section is a direct consequence of Proposition 6.1 and Theorem 7.1. In what follows, we provide a justification for parts $2-5$ of the algorithm.

9.1. Justification of integration rules. Assumption (ii) in section 3 guarantees that there is a finite number of $t^{-}, t^{\partial}$ points in $K$. Assumption (iii) and the compactness of $K$ imply that there is a finite number of equilibria in $K$. Therefore, part 2 of the algorithm performs a finite number of integrations.

Proposition 6.1 and Theorem 7.1 identify which extremal arcs can be part of $\partial K^{\star}$. The integrations in Table 8.1 produce all these arcs. The only portion of the table that requires justification is the one with entries that read "do nothing." The justification is provided in the following lemma.

Lemma 9.1. Let $p$ be a $t^{\partial}$ point which is the tail (resp., head) of an invariant arc of $\partial K$ and is such that the vector $f_{R}(p)$ (resp., $f_{L}(p)$ ) is tangent to $\partial K$. Then, the $R$-arc through $p$ exits $K$ in both positive and negative time.

Proof. We will prove the lemma assuming, without loss of generality, that $f_{R}(p)$ is tangent to $\partial K$. We will denote by $\mathcal{K}$ the invariant arc of $\partial K$ of which $p$ is the tail. By assumption (vi) in section $3, p \notin \mathcal{L}$, and therefore the vectors $f_{L}(p), f_{R}(p)$ are not parallel. Since $f_{R}(p)$ is tangent to $\partial K, f_{L}(p)$ must point toward the interior of $K$. Since $p \notin \mathcal{L}$, the extremal vector fields are $C^{1}$ in a neighborhood of $p$. By continuity, for all $q \in \partial K$ near $p, f_{L}(p)$ points toward the interior of $K$. Therefore, if $q \in \partial K$ is near $p$ but is outside $\mathcal{K}$ (i.e., $q$ is not contained in an invariant arc of $\partial K$ ), it must be that $f_{R}(q)$ points toward the exterior of $K$. Moreover, by assumption (ii) in section 3 , $f_{R}(q)$ cannot be tangent to $\partial K$ for all $q \in \partial K$ near $p$ such that $q \neq p$, and so it points toward the interior of $\mathcal{K}$. In summary, $f_{R}$ enjoys the following three properties: (1) $f_{R}(p)$ is tangent to $\partial K$; (2) for all $q \in \mathcal{K}$ near $p, q \neq p, f_{R}(q)$ points toward the interior of $K$; (3) for all $q \in \partial K \backslash \mathcal{K}$ near $p, q \neq p, f_{R}(q)$ points toward the exterior of $K$. Since $f_{L}(p)$ points toward the interior of $\partial K$, i.e., to the left of $\partial K$ and, at the same time, it points to the right of $f_{R}(p)$, it must be that the vector $f_{R}(p)$ and the oriented tangent vector to $\partial K$ at $p$ are antiparallel. This fact and properties (1)-(3) are illustrated in Figure 9.1. The only situation compatible with the properties above is that the $\mathrm{R}$-arc through $p$ exits $K$ in both positive and negative time.

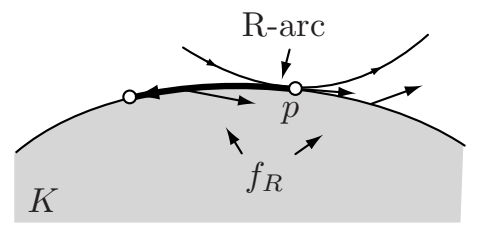

FIG. 9.1. Illustration of argument in the proof of Lemma 9.1. 
The stopping criteria for the integration derive from the following facts:

1. By Lemma 5.4, the only points in $\partial K^{\star} \cap \mathcal{L}^{-}$are $t^{-}$points.

2. By Proposition 5.3, $\partial K^{\star} \cap \partial K$ is made of invariant arcs of $\partial K$.

3. We are not interested in extremal arcs outside of $K$.

4. If an extremal arc originating in $K$ does not cross $\partial K$ or $\mathcal{L}^{-}$, then by Lemma 4.1 uniqueness of solutions (in backward and forward time) holds along the entire arc and by Theorem 5 on p. 139 of [16] the arc can approach (in finite or infinite time) an equilibrium or a compact limit set.

In light of the above, the integration step of the algorithm is guaranteed to terminate.

9.2. Justification of pruning rules. The partitioning of extremal arcs in step 3.1 guarantees that the head and tail of each subarc are significant points, and no other significant point is contained in the subarc. Arcs that spiral around limit sets are an exception, in that the head or tail of these subarcs are not well-defined.

The pruning, in step 3.2, of pairs of L- and R-arcs whose endpoints coincide is justified in Lemma 9.2 below.

The pruning, in step 3.3, of an L-arc with head at a point $p$ which is not special and where no L-arc has a tail follows from Proposition 6.1. Specifically, since $p$ is not special, the L-arc can only concatenate as $\mathrm{L} \stackrel{p}{\rightarrow} \rightarrow \mathrm{L}$, but such concatenation is not available. Hence, the L-arc in question cannot be part of the closed loop forming $\partial K^{\star}$. The same reasoning holds for R-arcs.

In step 3.4, the pruning of arcs whose head or tail is not connected to any other arc is motivated by the obvious observation that such arcs cannot be part of a closed loop and thus cannot be part of $\partial K^{\star}$.

The pruning in step 3.6 of any extremal arc $\gamma$ that spirals around a limit set is motivated by the following considerations. The partitioning of arcs in step 3.1 guarantees that $\gamma$ may only intersect other extremal arcs or invariant arcs of $\partial K$ at one of its endpoints. Since it is not possible to form a closed loop with $\gamma$ without creating further intersections, $\gamma$ cannot be part of a closed loop forming $\partial K^{\star}$ and should be eliminated.

The elimination of isolated significant points in step 3.7 of the procedure is obvious. A significant point connected to only two arcs of the same type can be eliminated. The result is a larger arc resulting from the HT concatenation of the two arcs.

At the end of the pruning procedure there is only a finite number of significant points and extremal arcs left, as shown in Lemma 9.3 below.

Lemma 9.2. Suppose that an L-arc $\gamma$ and an $R$-arc $\eta$ enjoy these properties:

(i) They share the same endpoints.

(ii) They do not contain any special point.

(iii) The only significant points in $\gamma$ and $\eta$ are their endpoints.

Then, $\gamma$ and $\eta$ cannot be part of $\partial K^{\star}$.

Proof. The two arcs $\gamma$ and $\eta$ enclose a compact set $\mathcal{R}$. There are two cases.

Case 1. $\eta$ and $\gamma$ have the same tails and heads. In this case, $\mathcal{R}$ is positively or negatively invariant for $\Sigma$, depending on the orientation of the arcs. Suppose $\mathcal{R}$ is positively invariant for $\Sigma$. This situation is depicted in Figure 9.2(a). Then, either $\mathcal{R}=K^{\star}$ or $\mathcal{R} \subsetneq K^{\star}$. It cannot be that $\mathcal{R}=K^{\star}$ because in this case the boundary of $K^{\star}$ would violate Proposition 6.1 since $\eta$ and $\gamma$ would concatenate $\mathrm{HH}$ at a point which is not special. Therefore, it must be that $\mathcal{R} \subsetneq K^{\star}$. Since $\partial \mathcal{R}=\eta \cup \gamma$ and since $\eta$ and $\gamma$ do not contain special points, by Proposition 6.1 each of $\eta$ and $\gamma$ is either entirely contained in $\partial K^{\star}$ or its intersection with $\partial K^{\star}$ is empty. The former situation cannot occur for both $\gamma$ and $\eta$ because $\mathcal{R} \subsetneq K^{\star}$. Therefore, at least one of the arcs, say, $\gamma$, has empty intersection with $\partial K^{\star}$. We claim that the same holds for 

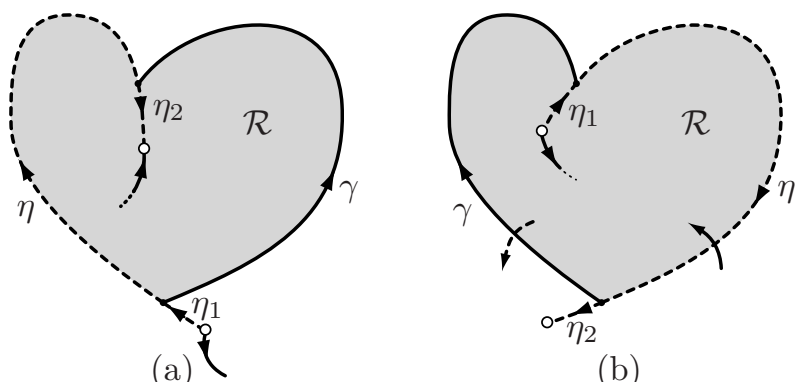

(b)

FIG. 9.2. Illustration of proof of Lemma 9.2.

$\eta$. In order that $\eta \subset \partial K^{\star}$, since the endpoints of $\eta$ are not special the only possible concatenations for $\eta$ are $\eta_{1} \rightarrow \rightarrow \eta \rightarrow \rightarrow \eta_{2}$, where $\eta_{1}$ and $\eta_{2}$ are R-arcs. Call $\bar{\eta}$ this new arc. The head of $\bar{\eta}$ can make an HH concatenation with an L-arc or an HT concatenation with another R-arc. In this latter case we can replace $\bar{\eta}$ by the larger $\mathrm{R}$-arc obtained after the HT concatenation. Therefore, the only case of interest is the $\mathrm{HH}$ concatenation with an L-arc. A similar argument holds for the tail of $\bar{\eta}$. In summary, we have the following properties, illustrated in Figure 9.2(a):

(a) $\mathcal{R}$ is positively invariant.

(b) L-arcs can enter $\mathcal{R}$ only through $\eta$, and R-arcs can enter $\mathcal{R}$ only through $\gamma$.

(c) The head of $\bar{\eta}$ is in $\mathcal{R}$, while the tail is outside $\mathcal{R}$.

(d) The head and tail of $\bar{\eta}$ can only make, respectively, HH and TT concatenations with two L-arcs.

The properties above and the constraints on concatenations imposed by Proposition 6.1 imply that in order for $\bar{\eta}$ to be part of a closed loop forming $\partial K^{\star}$, it is necessary that there is an L-arc in $\partial K^{\star}$ which enters $\mathcal{R}$ through a point in $\eta$ which is not an endpoint. This fact contradicts assumption (iii) of the lemma. The argument in the case when $\mathcal{R}$ is negatively invariant is analogous.

Case 2. The tail of $\gamma$ is the head of $\eta$, and vice versa. In this case, $\gamma$ and $\eta$ cannot be concatenated and thus $\mathcal{R} \neq K^{\star}$. As before, at most one of these arcs is a subset of $\partial K^{\star}$, say, $\eta$, and the only available concatenation for $\eta$ is $\eta_{1} \rightarrow \rightarrow \eta \rightarrow \rightarrow \eta_{2}$, where $\eta_{1}$ and $\eta_{2}$ are R-arcs, giving rise to a new R-arc $\bar{\eta}$. Depending on the orientation of $\eta$ and $\gamma$, only R-arcs may exit (resp., enter) $\mathcal{R}$, and they do so through $\gamma$, and only L-arcs may enter (resp., exit) $\mathcal{R}$, and they do so through $\eta$. One of these two situations is depicted in Figure 9.2(b). In order for $\bar{\eta}$ to be part of $\partial K^{\star}$ it is necessary that there is an arc entering or exiting $\mathcal{R}$ through a point in $\gamma$ or $\eta$ which is not an endpoint of these two arcs. This cannot occur by assumption (iii) of the lemma.

LEMma 9.3. The number of significant points and arcs left at the end of the pruning procedure is finite.

Proof. As argued earlier, part 2 of the algorithm produces a finite number of integrations. An infinite number of significant points can only arise from a finite number of integrations if some of these arcs produce infinitely many intersections. This can occur only if there exists at least one extremal arc $\gamma$, which without loss of generality we can assume to be an L-arc, which spirals toward a limit set, and there is another extremal arc $\eta$ crossing $\gamma$ as illustrated in Figure 9.3. In this case, the set $\gamma \cap \eta$ is a countably infinite sequence $\left\{p_{i}\right\}_{i \in \mathbb{N}}$ with limit point $p$. Without loss of generality, we may assume that $\gamma$ does not contain any equilibrium, $t^{-}$, or $t^{\partial}$ point, except possibly for the point $p$. Indeed, if $\gamma$ does contain such a point, we can replace $\gamma$ by a suitable subarc containing $p$. We can also assume that $p$ is an endpoint of $\eta$. 


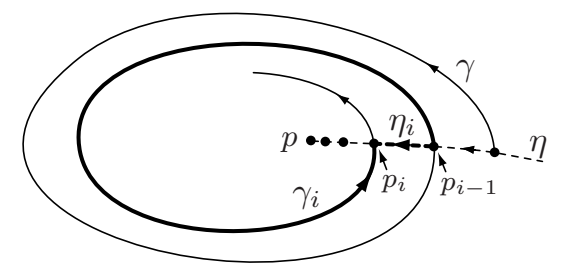

FIG. 9.3. Illustration of proof of Lemma 9.3.

Step 3.1 of the pruning procedure partitions $\gamma$ and $\eta$ into subarcs $\gamma_{i}, \eta_{i}$ with endpoints $p_{i}$ and $p_{i-1}, i \in \mathbb{N}$. The subarcs $\gamma_{i}$ and $\eta_{i}$ have their head at $p_{i}$ or $p_{i-1}$ depending on the orientation. Since the endpoints of $\gamma_{i}$ and $\eta_{i}$ coincide and are not special, step 3.2 of the procedure prunes them, and step 3.6 eliminates their endpoints. The outcome is that the infinite sequence $\left\{p_{i}\right\}_{i \in \mathbb{N}}$ is eliminated from the list of significant points and the $\operatorname{arcs} \gamma_{i}, \eta_{i}, i \in \mathbb{N}$, are pruned. Step 3.6 of the procedure eliminates the limit point $p$ from the list of significant points, unless some other arc has an endpoint there.

9.3. Justification of the graph construction rules. The graph construction rules ensure that any path in $\mathcal{G}$ corresponds to concatenations of arcs that are crossed consistently on one side by solutions of $\Sigma$. This is because the edges associated with $\mathrm{R}$-arcs are assigned opposite orientation to that of the edges associated with L-arcs and invariant $\operatorname{arcs}$ of $\partial K$. The orientation of edges of $\mathcal{G}$ and the association to every significant point in $\mathcal{P}$ which is not special of two vertices $v_{p}^{L}, v_{p}^{R}$ with an edge from $v_{p}^{R}$ to $v_{p}^{L}$ guarantees that any path in $\mathcal{G}$ corresponds to a valid concatenation on $\partial K^{\star}$, i.e., one satisfying the rules of Proposition 6.1.

Concatenations at special points can only be made as follows:

(a) $\mathrm{HH}$ concatenations of arcs of different type $(\mathrm{L} \rightarrow \leftarrow \mathrm{R}, \partial K \rightarrow \leftarrow \mathrm{R})$,

(b) HT concatenations of arcs of the same type ( $\rightarrow \rightarrow \partial K, \partial K \rightarrow \rightarrow \mathrm{L}, \mathrm{L} \rightarrow \rightarrow \mathrm{L}$, $\mathrm{R} \rightarrow \rightarrow \mathrm{R}$ ),

(c) TT concatenations of arcs of different type $(\partial K \leftarrow \rightarrow R, L \leftarrow \rightarrow R)$.

It is obvious that all constraints above are fulfilled by reversing the direction of edges associated with R-arcs. On the other hand, at significant points that are not special, one can only have TT concatenations $\partial K \leftarrow \rightarrow \mathrm{R}$ and $\mathrm{L} \leftarrow \rightarrow \mathrm{R}$ or HT concatenations $\partial K \rightarrow \rightarrow \mathrm{L}$, so certain concatenations must be disallowed. The possible intersections at such significant points are depicted in Figure 9.4. The left-hand side of the figure illustrates arcs intersecting at a point on $\partial K$. The only feasible concatenations, $\mathrm{A} \rightarrow \stackrel{p}{\rightarrow} \rightarrow \mathrm{B}$, $\mathrm{A} \stackrel{p}{\rightarrow} \rightarrow \mathrm{D}, \mathrm{C} \stackrel{p}{\leftarrow} \rightarrow \mathrm{B}, \mathrm{C} \stackrel{p}{\leftarrow} \rightarrow \mathrm{D}$, are precisely all possible paths of the associated graph. The same holds for the right-hand side of the figure, which depicts a significant point in int $K$. In this case, the feasible concatenations are $\mathrm{A} \stackrel{p}{\rightarrow} \mathrm{B}, \mathrm{C} \rightarrow \stackrel{p}{\rightarrow} \mathrm{D}, \mathrm{D} \stackrel{p}{\leftarrow} \rightarrow \mathrm{B}$. In particular, the concatenation $\mathrm{A} \rightarrow \stackrel{p}{\leftarrow} \leftarrow \mathrm{C}$ is not allowed because $p$ is not a special point, and indeed there is no corresponding path in the graph.

9.4. Justification of cycle analysis. The elimination in step 5.2 of any cycles containing two nonconsecutive vertices $v_{p}^{R}, v_{p}^{L}$ guarantees that all remaining simple cycles in $\mathcal{G}$ correspond to Jordan curves in the plane. Indeed, the only way that a simple cycle in $\mathcal{G}$ may correspond to a curve with a self-intersection is that the selfintersection occurs at a point $p$ which is associated with two vertices in $\mathcal{G}$. This only occurs when $p$ is a significant point which is not special and the cycle in $\mathcal{G}$ contains both $v_{p}^{L}$ and $v_{p}^{R}$. If $v_{p}^{R}, v_{p}^{L}$ are consecutive vertices in the graph, then the curve visits 

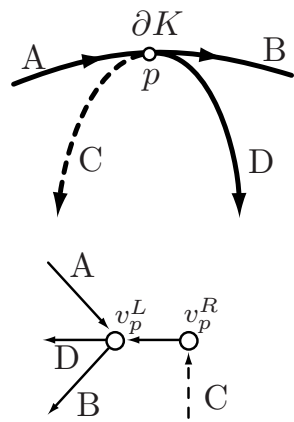
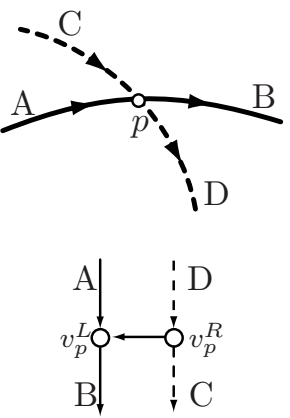

FIG. 9.4. Illustration of various arc intersections at significant points that are not special and their graph representation. Solid lines indicate arcs crossed leftward (L-arcs and invariant arcs of $\partial K)$, while dashed lines indicate $R$-arcs.
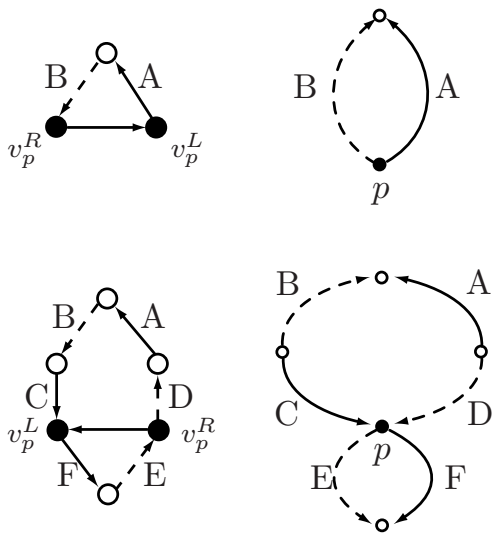

FIG. 9.5. Simple cycles in $\mathcal{G}$ with a pair of vertices $\left(v_{p}^{L}, v_{p}^{R}\right)$ and corresponding curves in the plane. The point $p$ is a significant point which is not special; all other points are special. The graph on the top left side has a cycle in which $v_{p}^{R}$ and $v_{p}^{L}$ are consecutive vertices. The corresponding curve on the top right side is Jordan. The graph on the bottom left side has a cycle in which $v_{p}^{R}$ and $v_{p}^{L}$ are not consecutive vertices (ABCFED). The corresponding curve on the bottom right side is not Jordan.

$p$ only once, and hence it has no self-intersections. Otherwise, the curve visits $p$ more than once and has self-intersections. Figure 9.5 illustrates the difference between a simple cycle with two consecutive vertices $v_{p}^{R}, v_{p}^{L}$ and one where these two vertices are nonconsecutive. Only in the former case is the closed curve Jordan.

As mentioned earlier, any path in $\mathcal{G}$ corresponds to a curve in the plane which is crossed consistently on one side. Hence, the Jordan curves associated with simple cycles of $\mathcal{G}$ delimit regions that are either positively or negatively invariant. After excluding in step 5.3 all the negatively invariant regions, we are left with a collection of positively invariant regions with the following properties:

(a) By construction, all regions are positively invariant and contained in $K$. Therefore, their union is contained in $K^{\star}$.

(b) By Theorem 7.1, any connected component of $\partial K^{\star}$ must be a concatenation of arcs forming a closed loop or a closed extremal arc. Since all such arcs are produced by the algorithm, any concatenation of arcs forming a closed loop in $\partial K^{\star}$ must correspond to a cycle in $\mathcal{G}$. In conclusion, $K^{\star}$ must be contained in the union of regions in step 5.4 of the algorithm. 
The considerations above lead to the conclusion that the algorithm produces the invariance kernel $K^{\star}$.

10. Example. Consider the planar system $\dot{x}=\lambda(t) f_{1}(x)+[1-\lambda(t)] f_{2}(x)$, where

$$
f_{1}(x)=\left[\begin{array}{c}
x_{2} \\
x_{1}^{2}+x_{1} x_{2}-1
\end{array}\right], \quad f_{2}(x)=\left[\begin{array}{c}
-x_{1}+2 x_{1}^{2} x_{2} \\
-3 x_{2}
\end{array}\right] .
$$

Let $K$ be the box $\left\{\left(x_{1}, x_{2}\right):\left|x_{1}\right| \leq 2,\left|x_{2}\right| \leq 2\right\}$ with rounded corners displayed in Figure 10.1. The corners are rounded to meet the standing assumptions in section 3 , but the invariance kernel algorithm can be applied with no modification even when $K$ has $C^{0}$ boundary.

The phase portraits of $f_{1}$ and $f_{2}$ are displayed in Figure 10.1. The vector field $f_{1}$ has two equilibria, a stable focus at $(-1,0)$ and a saddle at $(1,0)$, while $f_{2}$ has only one equilibrium at $(0,0)$, a stable node. The stable focus is not considered to be a special point. The collinearity set $\mathcal{L}$ and the phase portraits of the extremal vector fields $f_{L}$ and $f_{R}$ are displayed in Figure 10.2. The figure also displays all special points: two equilibria, one $t^{-}$point, and six $t^{\partial}$ points.
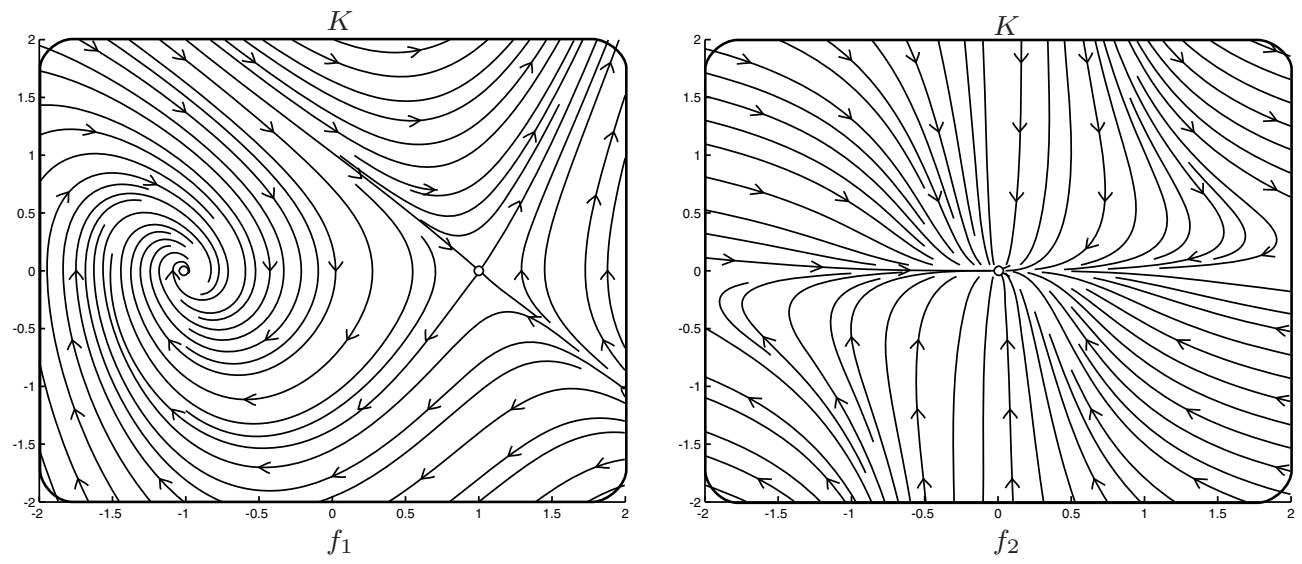

FIG. 10.1. Phase portraits of the vector fields $f_{1}$ and $f_{2}$. Circles denote equilibria.
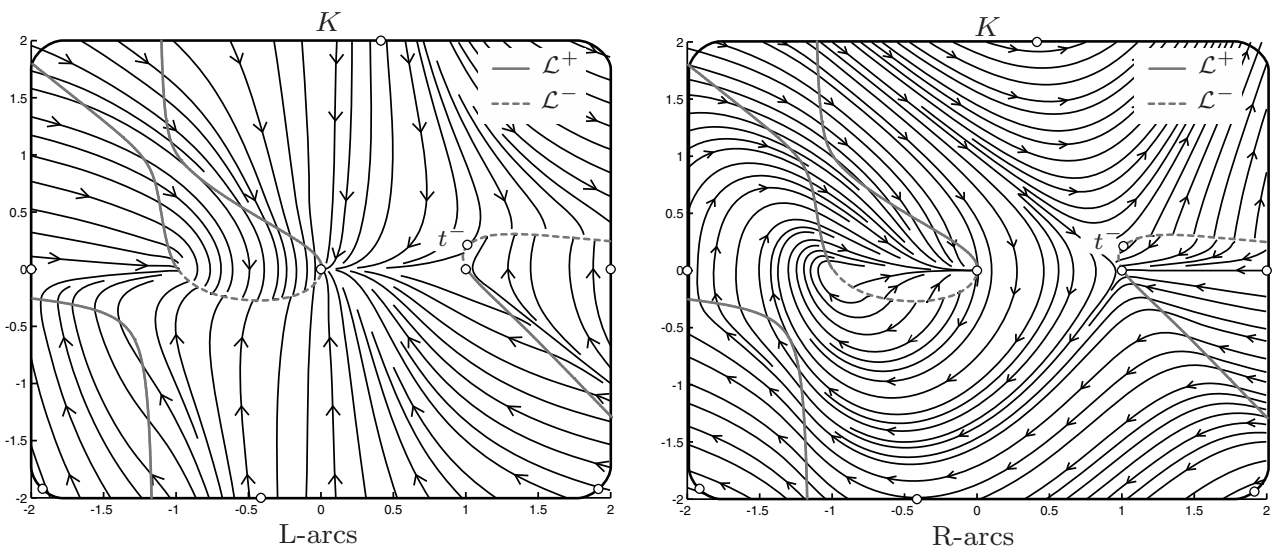

FIG. 10.2. The collinearity set $\mathcal{L}$ and phase portraits of the extremal vector fields $f_{L}$ and $f_{R}$. Circles denote special points: nodes, saddles, $t^{-}$, and $t^{\partial}$ points. 


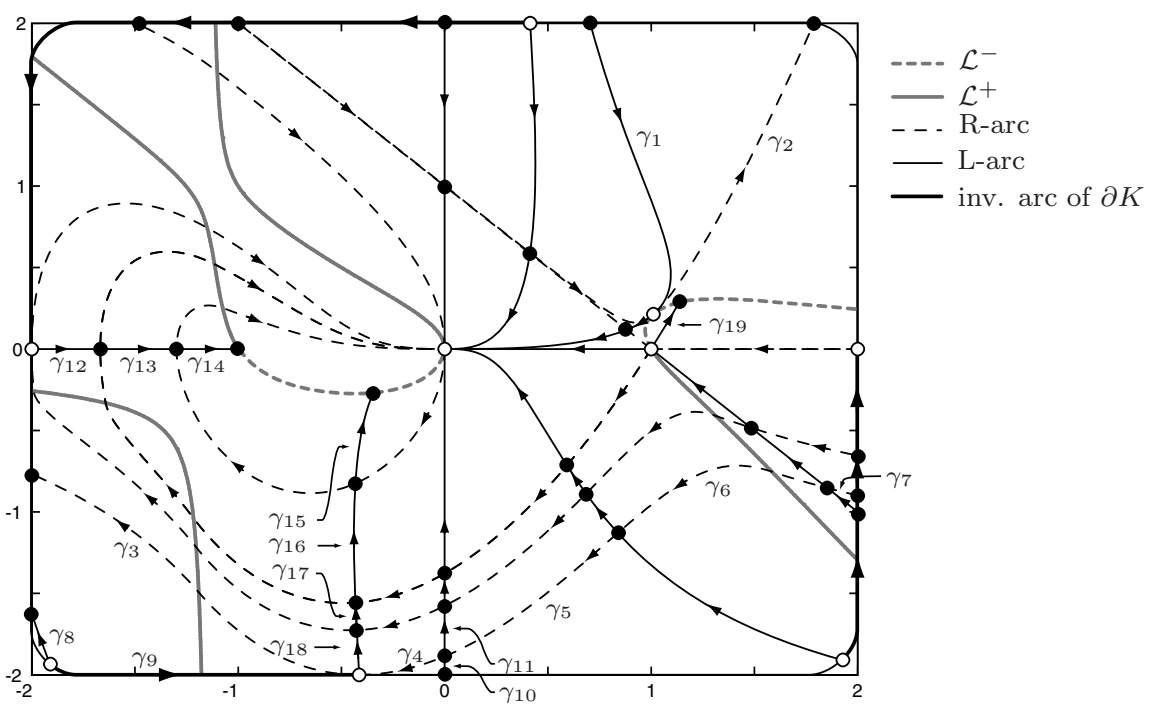

FIG. 10.3. Outcome of part 2 (integration) of the invariance kernel algorithm. Arcs $\gamma_{1}, \ldots, \gamma_{19}$ will be pruned in part 2 of the algorithm.

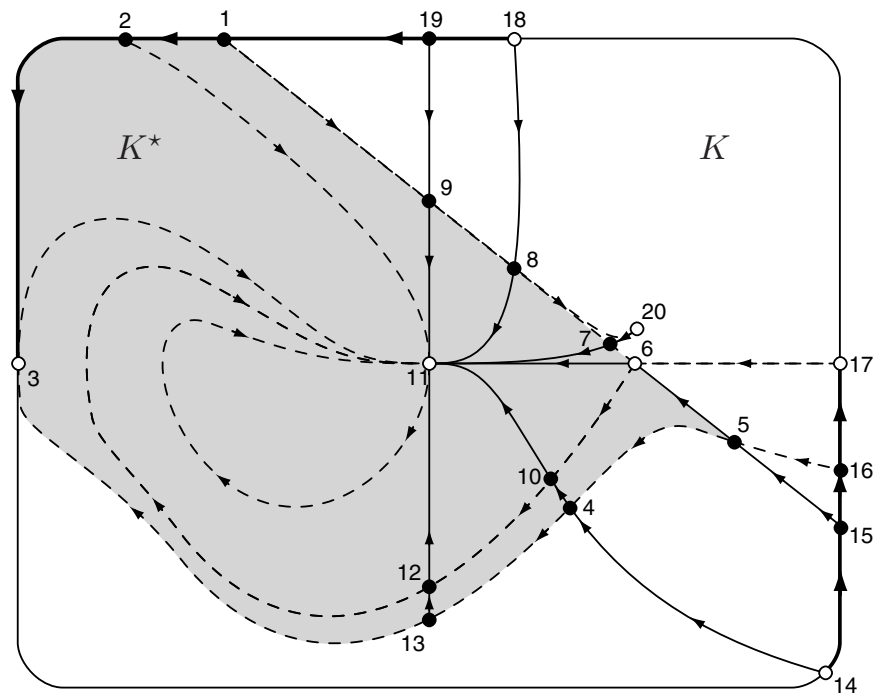

FIG. 10.4. Outcome of part 3 (pruning) of the invariance kernel algorithm. Shaded area is the invariance kernel $K^{\star}$.

The outcome of the integration part of the algorithm is displayed in Figure 10.3. Solid dots in the figure indicate all significant points arising from endpoints of integration and intersections of various arcs.

The outcome of the pruning part of the algorithm is displayed in Figure 10.4, where the $\operatorname{arcs} \gamma_{1}, \ldots, \gamma_{19}$ have been pruned in five executions of steps 3.3 and 3.4 of the algorithm as follows:

Copyright $(\odot$ by SIAM. Unauthorized reproduction of this article is prohibited. 


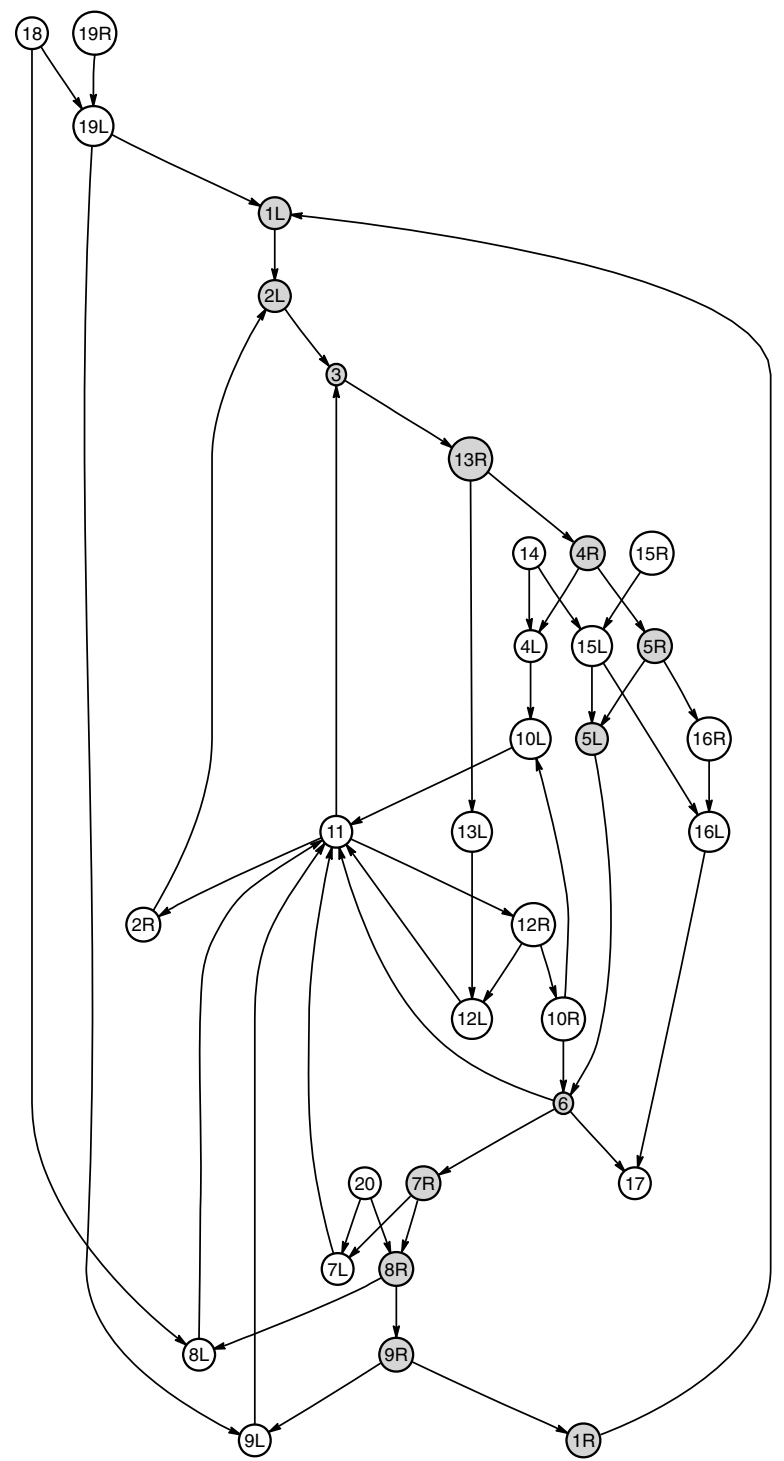

FIG. 10.5. Outcome of part 4 (graph construction) of the invariance kernel algorithm. The simple cycle corresponding to $\partial K^{\star}$ is highlighted with shaded nodes.
ExECUTION 1: $\quad$ STEP 3.3: $\gamma_{2}, \gamma_{3}, \gamma_{8}, \gamma_{14}, \gamma_{15}, \gamma_{19}$
ExeCution 2: $\quad$ SteP 3.3: $\gamma_{4}, \gamma_{13}, \gamma_{16}$
ExeCuTion 3: $\quad$ SteP 3.3: $\gamma_{5}, \gamma_{12}, \gamma_{17}$
EXECUTION 4: STEP 3.3: $\gamma_{6}, \gamma_{18}$
Execution 5: SteP 3.3: $\gamma_{7}$
STEP 3.4: $\gamma_{1}, \gamma_{9}, \gamma_{10}$
STEP 3.4: NO ACTION
STEP 3.4: $\gamma_{11}$
STEP 3.4: NO ACTION
STEP 3.4: NO ACTION

As a result of this pruning, in step 3.7 a number of significant points with no arcs attached or points connecting only two arcs of the same type are eliminated.

In part 4 of the algorithm we construct the invariance graph $\mathcal{G}$. It has 33 nodes and 49 edges and is displayed in Figure 10.5. For brevity, we will not list all cycles of $\mathcal{G}$, but the cycle corresponding to the boundary of the invariance kernel is highlighted in the figure. There is only one closed extremal arc in $K$, namely, the dashed curve 
containing point 11 in Figure 10.4. The resulting invariance kernel $K^{\star}$ is the shaded area in Figure 10.4.

11. Conclusions. The theory and algorithm presented in this paper are applicable only to planar single-input systems. There is no hope to extend our results to higher dimensional systems because our methodology heavily relies on a number of features that are present only in dimension two. On the other hand, we surmise that our results can be easily adapted to the problem of determining viability kernels. It should be possible to extend the theory to the case when the state space is a two-dimensional manifold and when $K$ is not simply connected.

\section{REFERENCES}

[1] A.A. Agrachev and D. Liberzon, Lie-algebraic stability criteria for switched systems, SIAM J. Control Optim., 40 (2001), pp. 253-269.

[2] J.-P. Aubin And A. Cellina, Differential Inclusions: Set-Valued Maps and Viability Theory, Springer, Berlin, 1984.

[3] J.-P. AuBin, Viability Theory, Birkhäuser Boston, Cambridge, MA, 1991.

[4] J.-P. Aubin, Viability kernels and capture basins of sets under differential inclusions, SIAM J. Control Optim., 40 (2001), pp. 853-881.

[5] M.M. Baitman, Controllability regions in the plane, M.M Differents. Uravn, 14 (1978), pp. 407417.

[6] G. Basile and G. Marro, Controlled and conditioned invariant subspaces in linear system theory, J. Optim. Theory Appl., 3 (1969), pp. 306-315.

[7] F. Blanchini, Set invariance in control, Automatica, 35 (1999), pp. 1747-1767.

[8] N. Bonneull and K. Mullers, Viable populations in prey-predator system, J. Math. Biol., 35 (1997), pp. 261-293.

[9] U. Boscain, G. Charlot, and M. Sigalotti, Stability of planar nonlinear switched systems, Discrete Contin. Dyn. Syst., 15 (2006), pp. 415-432.

[10] M.E. Broucke AND J. TuRrifF, Viability kernels for nonlinear control systems using bang controls, IEEE Trans. Automat. Control, 55 (2010), pp. 1280-1284.

[11] N.N. Butenina, Immunity zones of controlled dynamical systems, Differential Equations, 35 (1999), pp. 634-641.

[12] F.H. Clarke, Yu. S. Ledyaev, R.J. Stern, and P.R. Wolenski, Nonsmooth Analyisis and Control Theory, Springer, Berlin, 1991.

[13] A.A Davydov, Qualitative Theory of Control Systems, American Mathematical Society, Providence, RI, 1994.

[14] L. Doyen and C. Bene, Sustainability of fisheries through marine reserves: A robust modeling analysis, J. Environmental Management, 69 (2003), pp. 1-13.

[15] L. Doyen, M. De Lara, J. Ferraris, and D. Pelletier, Sustainability of exploited marine ecosystems through protected areas: A viability model and a coral reef case study, Ecological Modelling, 208 (2007), pp. 353-366.

[16] A.F. FILIPPov, Differential Equations with Discontinuous Righthand Sides, Kluwer Academic, Dordrecht, The Netherlands, 1988.

[17] H. Frankowska AND M. Quincampoix, Viability kernels of differential inclusions with constraints: Algorithm and applications, J. Math. Syst. Estimation Control, 1 (1991), pp. 371388.

[18] A. Isidori, Nonlinear Control Systems, 3rd ed., Springer, New York, 1995.

[19] I. Kolmanovsky and E. Gilbert, Theory and computation of disturbance invariant sets for discrete-time linear systems, Math. Problems Engrg., 4 (1998), pp. 317-367.

[20] G. Labinaz And M. GuAy, Viability, the solution set, and fixed point approximation of hybrid systems, Internat. J. Hybrid Systems, 3 (2003), pp. 189-216.

[21] M. Margaliot AND D. Liberzon, Lie-algebraic stability conditions for nonlinear switched systems and differential inclusions, Systems Control Lett., 55 (2006), pp. 8-16.

[22] V. Martinet And L. Doyen, Sustainability of an economy with an exhaustible resource: A viable control approach, Resource and Energy Economics, 29 (2007), pp. 17-39.

[23] M. Quincampoix, Differential inclusions and target problems, SIAM J. Control Optim., 30 (1992), pp. 324-335. 
[24] S.V. Rakovic, E. Kerrigan, K. Kouramas, And D.Q. Mayne, Invariant approximations of the minimal robust positively invariant set, IEEE Trans. Automat. Control, 50 (2005), pp. 406-410.

[25] B.G. Rawn, Ensuring Safe Exploitation of Wind Turbine Kinetic Energy: An Invariance Kernel Formulation, Ph.D. thesis, University of Toronto, 2009.

[26] J. Rieger, Shadowing and the viability kernel algorithm, Appl. Math. Optim., 60 (2009), pp. 429-441.

[27] P. SAint-PierRe, Approximation of the viability kernel, Appl. Math. Optim., 29 (1994), pp. 187-209.

[28] R. TARJAN, Enumeration of the elementary circuits of a directed graph, SIAM J. Comput., 2 (1973), pp. 211-216.

[29] W.M. WONHAm AND A.S. Morse, Decoupling and pole assignment in linear multivariable systems: A geometric approach, SIAM J. Control Optim., 8 (1970), pp. 1-18.

Copyright $@$ by SIAM. Unauthorized reproduction of this article is prohibited. 